\title{
Title: Elimination of HSV-2 infected cells is mediated predominantly by paracrine effects of tissue-resident $T$ cell derived cytokines
}

One Sentence Summary: Control of herpes simplex virus-2 is primarily mediated by rapidly diffusing cytokines secreted by tissue-resident $\mathrm{T}$ cells.

Authors: Pavitra Roychoudhury ${ }^{1,3}$, David A Swan ${ }^{1}$, Elizabeth Duke ${ }^{1,4}$, Lawrence Corey ${ }^{1,3,4}$, Jia Zhu $^{1,3}$, Veronica Davé ${ }^{1,6}$, Laura Richert Spuhler ${ }^{1}$, Jennifer M. Lund ${ }^{1,6}$, Martin Prlic ${ }^{1,6}$, Joshua T. Schiffer*1,2,4

\section{Affiliations:}

${ }^{1}$ Vaccine and Infectious Disease Division, Fred Hutchinson Cancer Research Center, Seattle, WA, 98109.

${ }^{2}$ Clinical Research Division, Fred Hutchinson Cancer Research Center, Seattle, WA, 98109.

${ }^{3}$ Department of Laboratory Medicine, University of Washington, Seattle, WA, 98195.

${ }^{4}$ Department of Medicine, University of Washington, Seattle, WA, 98195.

${ }^{5}$ Department of Immunology, University of Washington, Seattle, WA, 98109.

${ }^{6}$ Department of Global Health, University of Washington, Seattle, WA, 98104.

*corresponding author, jschiffe@,fredhutch.org 


\begin{abstract}
:
The mechanisms underlying rapid elimination of herpes simplex virus-2 (HSV-2) in the human genital tract despite low tissue-resident CD8+ T-cell density $\left(\mathrm{T}_{\mathrm{RM}}\right)$ are unknown. We analyzed shedding episodes during chronic HSV-2 infection: viral clearance always occurred within 24 hours of detection even if viral load exceeded $10^{7}$ HSV DNA copies; surges in granzyme B and interferon- $\gamma$ occurred within the early hours after reactivation. We next developed a mathematical model of an HSV-2 genital ulcer to integrate mechanistic observations of $\mathrm{T}_{\mathrm{RM}}$ in situ proliferation, trafficking, cytolytic effects and cytokine alarm signaling from murine studies with viral kinetics, histopathology and lesion size data from humans. A sufficiently high density of HSV-2 specific $\mathrm{T}_{\mathrm{RM}}$ predicted rapid contact-mediated elimination of infected cells. At lower $\mathrm{T}_{\mathrm{RM}}$ densities, $\mathrm{T}_{\mathrm{RM}}$ must initiate a rapidly diffusing, polyfunctional cytokine response in order to eliminate of a majority of infected cells and eradicate briskly spreading HSV-2 infection.
\end{abstract}




\section{[Main Text: ]}

\section{Introduction}

Tissue resident memory CD8+ $\mathrm{T}$ cells $\left(\mathrm{T}_{\mathrm{RM}}\right)$ rapidly identify and control recurrent human viral infections within peripheral sites (1-11). $\mathrm{T}_{\mathrm{RM}}$ lodge preferentially at prior sites of pathogen replication without recirculating in blood, and are differentiated from circulating memory CD8+ T cells based on micro-anatomic localization, cell-surface markers, and transcriptional profile (5, 12-15). During HSV-2 infection in humans, cells with $\mathrm{T}_{\mathrm{RM}}$ characteristics cluster at the dermal-epidermal junction where neuron endings release HSV-2 into the mucosa $(9,10,16)$. The heterogeneous spatial aggregation of $\mathrm{T}_{\mathrm{RM}}$ in genital skin explains the considerable variability of HSV-2 severity, including episodes which are contained after a few hours of viral replication and those that persist for many days and cause symptomatic lesions (17-21).

Recent murine studies employing intravital imaging describe the extraordinary range of $\mathrm{T}_{\mathrm{RM}}$ control mechanisms in situ. In the absence of antigen, $\mathrm{T}_{\mathrm{RM}}$ efficiently survey previously infected tissues to recognize and eliminate infected cells during re-infection $(1,22)$. During this immunosurveillance phase, $\mathrm{T}_{\mathrm{RM}}$ express dendritic-like arms to contact a large number of potentially infected cells $(1,22)$, and remain in an activated state despite the absence of antigen-driven stimulus via the T-cell receptor $(8)$. Upon recognition of an infected cell, $\mathrm{T}_{\mathrm{RM}}$ motility is restricted and abundant local proliferation ensues (22). $\mathrm{T}_{\mathrm{RM}}$ then mediate efficient, contact-mediated apoptosis of infected cells by releasing perforin and granzyme B (23-25).

Upon activation, $\mathrm{T}_{\mathrm{RM}}$ induce a broad antiviral program consisting of cytokine release and resultant generalized local inflammation $(1,8)$. Cytokines, notably interferon- $\gamma($ IFN- $\gamma)$, induce clearance of infected cells $(26,27)$, and render surrounding cells resistant to infection, even with unrelated pathogens (1). Chemokines such as CXCL9 and 10 may draw local immune cells to infected 
sites $(11,28)$. Cytokines and chemokines diffuse rapidly, are efficiently internalized by their target cells (29) and exert paracrine effects upon cells that are not in direct contact with $\mathrm{T}_{\mathrm{RM}}$.

Analysis of human biopsy specimens of HSV-2 infected tissues reveals high-density clusters of $\mathrm{T}_{\mathrm{RM}}$ in certain micro-regions, with low densities of $\mathrm{T}_{\mathrm{RM}}$ in others (20). Yet, a majority of HSV-2 shedding episodes are eliminated within 6-24 hours (19,21, 30). Because individual HSV-2 ulcers are small, this rapid peak in viral load is more likely due to an intense local immune response rather than target cell limitation $(19,30-32)$.

It is unknown how low numbers of $\mathrm{T}_{\mathrm{RM}}$ orchestrate elimination of infected cells so efficiently. Different mechanisms may mediate elimination of a few infected cells over hours during mild episodes, versus thousands of infected cells over days during more severe episodes. Here, we construct an agentbased mathematical model to recapitulate the spatiotemporal dynamics underlying the viral spread, cytokine expansion and $\mathrm{T}_{\mathrm{RM}}$-mediated containment of HSV-2 in genital tissues. 


\section{Results}

Intense immune pressure within 24 hours of $\mathrm{HSV}-2$ detection. A characteristic of HSV-2 shedding episodes in chronically infected persons is rapid viral expansion, followed by a slightly more protracted clearance phase $(21,33,34)$. While most episodes share this core feature, they vary in severity. Many asymptomatic episodes are cleared in 6-12 hours and peak at $<10^{4}$ HSV DNA copies. Other episodes exceed $10^{7} \mathrm{HSV}$ DNA copies and are associated with visible lesions and shedding over days to weeks $(30,35,36)$. In episodes longer than four days, longevity is driven by a rebound of HSV after an initial phase of clearance, possibly due to seeding of new ulcers in immunologically distinct microenvironments (19).

To approximate viral dynamics within single infection micro-environments, we re-analyzed 83 episodes (655 total swabs from 20 subjects) from a dataset in which study participants performed swabs every 6 hours over 60 days. We binned episodes by duration into short ( $<1$ day), medium (1-2 days), and long (>2 days) (30), and identified brisk viral expansion with an early peak in all episodes, even those with high viral loads (Fig. 1a). There was a wide distribution of peak viral loads (Fig. 1b) ranging from 2.2 to $7.9 \log 10 \mathrm{HSV}$ DNA copies. Episodes were of variable duration, but elimination usually occurred in less than 48 hours, with a minority lasting $>100$ hours (Fig. 1c). Median time to the first viral load peak was 7 hours; all episodes reached their first peak within 24 hours (Fig. 1d). Moderate correlations were noted between time to first peak and peak viral load (Fig. 1e), and time to first peak and duration (Fig. 1f).

These results confirm that massive HSV-2 replication is a defining early feature of shedding episodes. However, immunologic responses predominate within 20 hours of viral detection, and time of the initial peak predicts severity. Whether an episode progresses to high viral loads and days of shedding 
or rapid containment in the first several hours may depend on the intensity of the immune response during the early hours of reactivation.

\section{Heterogeneous dispersion of tissue-resident CD8+T cells $\left(T_{R M}\right)$ in HSV-2 infected genital tissues.}

HSV-2 reactivates in specific micro-environments throughout the human genital tract because of random travelling of virions along highly arborized branches of peripheral neurons (37). We observed a fixed spatial meta-structure of CD8+ T-cell density in infected tissue, defined by dense clusters in some regions and low numbers in others (20). To characterize tissue micro-environments, we summarized $\mathrm{T}_{\mathrm{RM}}$ densities observed in 19 genital biopsy specimens (Fig. 2a) performed at 2 and 8 weeks following lesion healing (We refer to all observed CD8 $+\mathrm{T}$ cells as $\mathrm{T}_{\mathrm{RM}}$ though it is possible that a minority are not truly resident). We divided each image into regions with 100 epidermal cells per region (Fig. 2b-c) and calculated the ratio of $\mathrm{T}_{\mathrm{RM}}$ to epidermal cells (in situ effector:target or $\mathrm{E}: \mathrm{T}$ ratio). We then created a rank-order distribution of all observed ratios (Fig. 2d). The median E:T ratio in the 1008 micro-regions was 0.02 (IQR 0.07, range $0-2$ ). $\mathrm{T}_{\mathrm{RM}}$ were absent in $26.2 \%$ of the micro-regions $(\mathrm{E}: \mathrm{T}=0)$. Median $\mathrm{E}: \mathrm{T}$ ratios for the 19 specimens ranged from 0.01 to 0.24 (IQR of medians $=0.02$ ). Exponential slopes of rank order abundance curves (median -0.121, IQR 0.048, range: -0.222 to -0.045) were similar across specimens (Fig. 2d), implying an equivalent gradient between high and low $\mathrm{T}_{\mathrm{RM}}$ density regions (Fig. 2a, c). Specimens with a higher proportion of micro-regions with $\mathrm{E}: \mathrm{T}=0$ had lower overall E:T ratios (Fig. 2e).

A mathematical model of $\mathrm{HSV}-2$ replication and spread within a single ulcer. We next sought to explain how infected cells are eliminated quickly, given the low $\mathrm{T}_{\mathrm{RM}}$ densities commonly observed in infected samples. To approximate spatial conditions of a single infection micro-environment, we 
developed a two-dimensional stochastic, agent-based mathematical model. Our goal was to precisely link spatial heterogeneity of $\mathrm{T}_{\mathrm{RM}}$ density (Fig. 2) with observed heterogeneity in shedding episode kinetics (Fig. 1). We developed the model in an iterative fashion to limit required parameter fitting at each step.

First, we simulated HSV-2 replication and spread assuming no $\mathrm{T}_{\mathrm{RM}}$ immunity (Fig. 3a, Materials and Methods for details and Table 1 for model parameters), to recapitulate early HSV-2 shedding episode kinetics. The agent-based model assumes that HSV-2 replication occurs in a two-stage process within an infected cell. Newly infected cells enter a pre-productive phase, during which immediate-early and early viral proteins are expressed but HSV DNA replication has yet to occur (38). After several hours, HSV DNA polymerase activity initiates, leading to production of infectious viral particles for $\sim 20$ hours until cell lysis and death (Fig. 3a).

Human HSV-2 infection occurs within nucleated epithelial cells in genital skin or mucosa. The three-dimensional anatomy of this tissue space comprises $\sim 2-10$ stacked epidermal cells between the surface of the skin and the dermal-epidermal junction where HSV-2 is released from nerve endings. For simplicity, our model comprises a two-dimensional lattice consisting of tightly packed square cells with 8 potential cellular contacts at edges and corners: this assumption is in accordance with the arrangement of squamous epithelial cells within tissue. Many cells in the top layers of human skin are not permissive to HSV replication, so lateral cell-to-cell spread of HSV-2 is more efficient than apical to basal spread (39). Nevertheless, by neglecting the vertical dimension of cells, the model may underestimate total number of infected cells and viral load.

To approximate the observation that infection of adjacent cells occurs via tight-junctions,(40) we allow individual viruses to diffuse with random directionality across the grid immediately after their 
production. Viruses that reside in the same model region as susceptible cells generate infection with a concentration-dependent probability at each time step (Materials and Methods).

Unimpeded $H S V-2$ spread in the absence of $\boldsymbol{T}_{\boldsymbol{R} M}$. In simulations without $\mathrm{T}_{\mathrm{RM}}$, viral load expands rapidly during the first 12-18 hours of infection (Fig. 3b), but reaches maximal values lower than those observed during the most severe shedding episodes (Fig. 1a,b). This discrepancy is likely due to our model's two-dimensional structure of cells. Moreover, the empirical data often include the sum of viral contributions from multiple ulcers, whereas we only model one ulcer (19).

In the absence of $\mathrm{T}_{\mathrm{RM}}$ activity, viral load does not peak but instead grows asymptotically with deceleration after one day (Fig. 3b). Both total number of infected cells (Fig. 3c) and ulcer diameter (Fig. 3d) expand in an unimpeded fashion. Realistic estimates of ulcer diameter (1-3 mm) are noted at 24-48 hours following infection with large ulcers after several days (Fig. 3d).

A two-dimensional structure of uncontrolled infection emerges from the model (Movie S1). The leading edge of simulated ulcers consists of pre-productive, newly infected cells, surrounding a thicker band of productively infected cells and a central core of dead and regenerating cells (Fig. 3e). An outer ring of high viral load is noted after two days (Fig. 3f) with a far outer ring of lower viral load due to viral diffusion. The asymptotic behavior of viral load relates to spatial features of the mathematical model: target cell limitation increases with further expansion of the simulated ulcer. Specifically, as infection spreads radially, new infected cells are increasingly likely to contact already infected or dead cells (41).

Persistent high viral load shedding and large non-healing ulcers are well-described features of infection in persons with severely compromised T cell immunity due to HIV/AIDS or stem cell 
transplantation(42). Our basic model recapitulates untreated HSV-2 infection in these clinical populations.

\section{A mathematical model incorporating $T_{R M}$ trafficking, dendricity, local proliferation, and rapid}

contact-mediated killing of infected cells. Most immunocompetent persons start to clear each HSV-2 shedding episode within a day after viral load peaks (Fig. 1). To capture this fact, we added $\mathrm{T}_{\mathrm{RM}}$ immunity to the baseline model (Materials and Methods, Table 2). For each simulated episode, we generated varying realistic densities of $\mathrm{T}_{\mathrm{RM}}$ across the modeled grid based on our analysis of biopsy specimens (Fig. 2), by randomly sampling rank abundance slope and $\mathrm{T}_{\mathrm{RM}}$ abundance within the highest ranked region (Fig. 2d). Forty percent of cultured CD8+ T cells isolated from HSV-2-infected tissue are activated in the presence of whole virus $(9)$; in our simulations, this percentage of $\mathrm{T}_{\mathrm{RM}}$ retains the ability to recognize HSV-2 antigen and kill adjacent infected cells in a contact-dependent manner.

Numerous recent murine studies allow parameterization of $\mathrm{T}_{\mathrm{RM}}$ behavior in tissue during the immunosurveillance phase between HSV-2 shedding episodes. When cognate antigen is not present, $\mathrm{T}_{\mathrm{RM}}$ patrol the cellular grid (Fig. 4a) (43). Recent evidence provides rates for $\mathrm{T}_{\mathrm{RM}}$ movement and suggests that trafficking follows a random walk over time scales of hours $(1,22)$. Patrolling $\mathrm{T}_{\mathrm{RM}}$ project dendritic arms to achieve a greater number of contacts with adjacent cells (Fig. 4a) $(1,22)$. We selected a value for $\mathrm{T}_{\mathrm{RM}}$ dendricity that captures the average number of target cells that a single $\mathrm{T}$ cell touches.

HSV-2 specific $\mathrm{T}_{\mathrm{RM}}$ undergo phenotypic changes upon contacting and engaging an infected cell, taking on a round morphology and transiently limited mobility. Brisk in situ $\mathrm{T}_{\mathrm{RM}}$ proliferation ensues, increasing $\mathrm{T}_{\mathrm{RM}}$ levels approximately 10-fold over a 24-hour period (Fig. 4a) (23, 24). Most local amplification of local $\mathrm{T}$ cell levels within 72 hours of antigen introduction is via $\mathrm{T}_{\mathrm{RM}}$ proliferation rather than trafficking of CD8+ T cells from blood or draining lymph nodes $(23,24)$. In most cases, HSV-2 
DNA has been eliminated from tissue at this point. Therefore, we included in situ $\mathrm{T}_{\mathrm{RM}}$ proliferation in our model, but excluded systemic trafficking of $\mathrm{T}$ cells.

Upon recognizing and adhering to an infected cell, $\mathrm{T}_{\mathrm{RM}}$ induce apoptosis within 30 minutes, presumably via secretion of granzyme B and perforin (Fig. 4a) (25). In our simulations, $\mathrm{T}_{\mathrm{RM}}$ occupy sites on the grid of epidermal cells. If $\mathrm{T}_{\mathrm{RM}}$ are at sites adjacent to (via a corner or edge), or in the same location as, an infected cell, they can kill these infected cells via TCR contact-mediated mechanisms at a fixed rate or in a synergistic fashion, such that an infected cell contacted by several $\mathrm{T}$ cells dies more rapidly (Materials and Methods) (25). We also assume that a single T cell can concurrently kill multiple adjacent infected cells via dendritic extensions (44). Cytokine paracrine effects against infected cells were not included in this version of the model.

\section{Unimpeded $H S V-2$ spread in the presence of $T_{R M}$ that exert contact-mediated killing but no paracrine}

effects. When we simulated the model with the above assumptions of $\mathrm{T}_{\mathrm{RM}}$ immunosurveillance and pathogen containment, there was insufficient immunologic pressure to eliminate all infected cells. Even at extremely high E:T ratios (>0.20) rarely observed in our specimens (Fig. 2e), only 21\% of 100 simulated episodes were contained in three days. We simulated 100 episodes at 10 realistic E:T ratios (range: 0.013-0.13). Asymptotic viral shedding was observed in all cases (Fig. 4b). E:T ratio was predictive of viral load setpoint in these simulations (Fig. 4c), indicating that contact-mediated killing by $\mathrm{T}_{\mathrm{RM}}$ exerts meaningful immunological pressure.

A spatial approximation of this infection model demonstrates that more infected cells are killed by $\mathrm{T}_{\mathrm{RM}}$ than direct viral lysis (Movie S2, Fig. 4d), and that viral loads are lower per cellular region over time (Movie S2, Fig. 4e), relative to simulations without $T_{R M}$ (Movie S1, Fig. 3e,f). Nevertheless, HSV2 spreads effectively through micro-regions of both low and high $\mathrm{T}_{\mathrm{RM}}$ density (Movie S3, Fig. 4f). We 
conclude that despite the addition of multiple $\mathrm{T}_{\mathrm{RM}}$ functions to our model, HSV-2 spread outpaces direct cell-to-cell killing via $\mathrm{T}_{\mathrm{RM}}$ and does not recapitulate observed shedding episode features in

immunocompetent humans (Fig. 1a). This observation led us to include non-contact mediated functions of $\mathrm{T}_{\mathrm{RM}}$ in our model.

Secretion of granzyme B and IFN-y early during human HSV-2 shedding episodes. To validate inclusion of cytokines in our mathematical model, we performed local sampling for cytokine quantitation every 3 hours during naturally occurring shedding episodes in persons with chronic HSV-2 infection. During all episodes, we noted an early 1-2 log surge in granzyme B (Fig. 5a, upper row) compatible with direct contact-mediated killing of infected cells by local T cells and NK cells. We detected an increase of similar magnitude in IFN- $\gamma$ concordant with episode peaks (Fig. 5a, lower row), suggesting that $\mathrm{T}_{\mathrm{RM}}$ exert paracrine effects. Because CD8+ T cells outnumber CD4+ T cells and NK cells in HSV-2 lesions at the dermal-epidermal junction (16), these cells are likely to be critical producers of both detectable granzyme B and IFN- $\gamma$ though we cannot rule out contribution from CD4+ T cells and NK cells in the dermis. Keratinocyte derived cytokines including IFN- $\alpha$ and IFN- $\beta$ did not consistently surge during these episodes and were often absent or present at much lower levels (Fig. S1a, b).

Model refinements to assess possible cytokine mechanisms for containment of local HSV-2 infection. $\mathrm{T}_{\mathrm{RM}}$-secreted cytokines may generate a local alarm state via numerous paracrine mechanisms after diffusing across a field of cells. We updated our model to include four potential cytokine functions: 1) enhanced elimination of infected cells, 2) reduction in viral replication rate, 3) reduction in HSV-2 infectivity and 4) induction of bystander (non HSV-specific) $\mathrm{T}_{\mathrm{RM}}$ proliferation and cytokine production 
(Fig. 5b). Inclusion of these mechanisms is experimentally justified: $\mathrm{T}_{\mathrm{RM}}$-derived cytokines, particularly IFN- $\gamma$, induce apoptosis in HSV-2 infected cells(27), and lower viral replication rate. IFN- $\gamma$ renders keratinocytes resistant to HSV-2 infection for several days in mice(2). Finally, cytokines may stimulate proliferation, differentiation and innate-like cytokine secretion from other local bystander CD8+ T cells in a paracrine fashion (45-49).

Because it is unknown which of these mechanisms are relevant during human infection, we generated 16 models with every possible combination, including a model lacking any cytokine functionality and one with all four functions. For all models, we assumed that bystander $\mathrm{T}_{\mathrm{RM}}$ are incapable of recognizing and lysing infected cells via direct contact.

We imputed experimentally observed values of cytokine production by activated $\mathrm{T}_{\mathrm{RM}}$ into the model (Table 3). The rate of cytokine diffusion across a cellular scaffold was estimated in vitro(29), and exceeds that of viral cell-to-cell spread. Given that rates of cellular uptake and diffusion of cytokines in vivo are unknown, we varied these two parameters across wide ranges of values for all models tested. For models including one or more of the mechanisms described above, we varied additional parameters. For instance, we included values to characterize the cytokine concentration at which a specific cytokine action was half-maximal in a given region.

We explored 1000 possible randomly selected parameter sets for the 16 models. For each unique parameter set, we simulated 100 episodes, each with different initial conditions of $\mathrm{T}_{\mathrm{RM}}$ density drawn randomly from exponential distributions (Fig. 2d). In total, we simulated 1,600,000 HSV-2 ulcers.

\section{Recapitulation of observed shedding episode kinetics with incorporation of HSV-2 specific $T_{R M}$}

cytokine secretion into the mathematical model. To identify which cytokine functions and parameters best recapitulate the observed data, we performed comparative model fitting to shedding episode data 
using Akaike Information Criteria (AIC), to reward for fit while penalizing for unnecessary complexity. We fit to shedding data from the 83 episodes described in Fig. 1. Episodes were arrayed in rank abundance curves according to peak viral load, duration and time to peak, such that the models were tasked with reproducing the entire distribution of shedding episode severity (Fig. S2). To mimic the clinical protocol, we assumed sampling every 6 hours and random episode initiation 0 to 6 hours after each simulated sample.

Optimal data fitting occurred with inclusion of all four cytokine mechanisms in Fig. 5b (Fig. S2a). We added a fifth possible mechanism to the model, cytokine induction of a chemical gradient to allow CD8+ T cells to home to sites of infection: this did not enhance data fitting and was not subsequently included. Similarly, adjusting modes of $\mathrm{T}_{\mathrm{RM}}$ trafficking from random walk to Levy walk did not alter model fit and was not subsequently included. These results suggest that elimination of HSV-2 infected cells by cytokines involves multiple complementary mechanisms.

The optimized model slightly overestimated distributions of episode peak viral load and durations at moderate ranks (Fig. S2b,c), underestimated peak viral load of the most severe episodes (Fig. S2b), but closely recapitulated distribution of time to episode peak (Fig. S2d). The model accepted a wide range of plausible values for rates of cytokine uptake into cells and viral diffusion. A narrower range was identified for cytokine diffusion rate, indicating the importance of this value for model fitting (Fig. S2e). Threshold upper limits were identified for necessary intracellular concentration of cytokines to limit viral replication and induce death in infected cells, prevent infection in uninfected cells, and activate surrounding $\mathrm{T}_{\mathrm{RM}}$. These values were low, suggesting that cells are extremely sensitive to these proteins (Table 3). 


\section{Rapid containment of infection under low $T_{R M}$ condition due to rapid cytokine diffusion derived from}

$\boldsymbol{H S V - 2}$ specific $\boldsymbol{T}_{\boldsymbol{R} M}$. Viral load trajectories in simulated episodes were notable for early peaks, rapid expansion rate and short duration (Fig. 5c); timing of cytokine surge (Fig. 5a, c) was comparable to human shedding episodes. As with murine studies (24), E:T ratio in the infected area was fairly predictive of peak viral load in simulations: this was true when a field of 15625 or 49 target cells was considered (Fig. 5d, e). A $T_{\mathrm{RM}}$ to epithelial cell ratio of roughly 0.2 was required for rapid elimination of HSV-2 infected cells prior to development of viral loads sufficient for lesion development and sexual transmission potential ( $>10^{4}$ HSV DNA copies) (Fig. 5d, e); this exceeded ratios observed in most human biopsy specimens (Fig. 2e). Early recognition of infection by $\mathrm{T}_{\mathrm{RM}}$ often resulted in elimination of virus in 6-24 hours with fewer than 20 total infected cells (Movie S4).

Spatial visualization demonstrates that in more severe episodes, later initial recognition of an infected cell by an HSV-2 specific $\mathrm{T}_{\mathrm{RM}}$ (due to a lower $\mathrm{T}_{\mathrm{RM}}$ density) is followed by rapid diffusion of cytokines and immediate impact of cytokine antiviral effects (Fig. 6a, Movies S5 \& S6). Accordingly, in simulations the time between infection of the first cell and recognition of an infected cell by an HSV2 specific $\mathrm{T}_{\mathrm{RM}}$ was predictive of peak viral load (Fig. 6b), number of infected cells (Fig. 6c) and episode duration (Fig. 6d). As episodes increased in severity, the proportion of infected cells killed by cytokine effects rather than direct lysis by $\mathrm{T}_{\mathrm{RM}}$ was generally higher (Fig. 6e).

\section{Polyfunctional and individually redundant antiviral cytokine effects within the HSV-2 infection}

micro-environment. To further delineate mechanisms of cytokine protection, we performed in silico knock out experiments by removing single and multiple cytokine mechanisms over 100 sequential shedding episodes. These simulations demonstrated that elimination of single cytokine mechanisms generally had a limited effect on episode severity; yet, when multiple cytokine mechanisms were not 
included in the model, a higher proportion of episodes had longer time to peak viral load (Fig. S3a), higher total number of infected cells (Fig. S3b), higher peak viral loads (Fig. S3c) and longer duration (Fig. S3d). Among cytokine mechanisms, activation of bystander $\mathrm{T}_{\mathrm{RM}}$ (particularly induction of cytokine secretion) and limitation of infected cell lifespan were most critical in limiting episode duration. Simulations in which cytokines did not impact infected cell lifespan (Movie S7) or HSV infectivity (Movie S8) led to slightly protracted episodes in which ulcers took on an uncharacteristic serpiginous appearance.

\section{More rapid HSV-2 elimination due to $T_{R M}$ trafficking, dendricity, proliferation, and cooperative}

killing. We performed additional in silico knock out experiments to identify the relative importance of non-cytokine mediated $\mathrm{T}_{\mathrm{RM}}$ mechanisms of immune control. Elimination of single immunosurveillance mechanisms generally had a limited effect on episode severity; when both $\mathrm{T}_{\mathrm{RM}}$ dendricity and mobility were eliminated from the model, a higher proportion of episodes had longer time to peak viral load (Fig. S4a), higher total number of infected cells (Fig. S4b), higher peak viral loads (Fig. S4c) and longer duration (Fig. S4d). Elimination of contact-mediated killing had no overall effect on episode severity, highlighting the potency of antiviral cytokine spread. 


\section{Discussion}

$\mathrm{T}_{\mathrm{RM}}$ play a critical role in mucosal protection against HSV-2. During chronic infection, genital tissue contains HSV-2 specific and bystander $\mathrm{T}_{\mathrm{RM}}$ months after local elimination of infected cells (9). The release of HSV-2 from latently infected ganglia via sensory neurons into genital tissues is temporally and spatially stochastic, resulting in seeding of low and high density $\mathrm{T}_{\mathrm{RM}}$ regions $(19,20,50)$. Within micro-regions, the spatial heterogeneity of $\mathrm{T}_{\mathrm{RM}}$ results in highly variable amounts of viral replication and subsequent $\mathrm{T}_{\mathrm{RM}}$-mediated expansion (21). At the whole tissue level, the result is a shedding pattern characterized by some brief episodes (approaching sterilizing immunity) in which infection is limited to a few cells, and other episodes that persist for days and are associated with lesions, infection of thousands of cells and high transmission risk $(51,52)$.

While HSV-2 achieves sufficient replication to induce a disease state in many infected people, the immune system wins each local battle with remarkable efficiency. Viral loads invariably peak within 24 hours after viral DNA detection; the abrupt transition from viral expansion to contraction is associated with a surge in granzyme B and IFN- $\gamma$. Using a mathematical model, we demonstrate that HSV-2 specific $T_{R M}$ induce rapid apoptosis of directly adjacent infected cells. If this occurs when infection is limited to one or a few cells, then the episode will terminate without need for an amplified local response. If the number of infected cells is greater, then $\mathrm{T}_{\mathrm{RM}}$ contact-mediated killing is insufficient for pathogen control, despite efficient $\mathrm{T}_{\mathrm{RM}}$ trafficking, expression of dendritic arms to canvas a high number of local cells, in situ proliferation, and rapid cooperative killing of infected cells.

The essential second step for truncating local shedding is cytokine secretion, which leads to a profound antiviral state in surrounding cells. Because they are smaller, secreted cytokines diffuse more rapidly than viruses, and decelerate the pace of HSV-2 spread (29). Based on best fit to observed data, our model suggests that cytokines exert distinct effects including rapid killing of infected cells, lowering 
HSV-2 replication, lowering HSV-2 infectivity to uninfected cells, and activating bystander $\mathrm{T}_{\mathrm{RM}}$. Experimental evidence suggests that IFN- $\gamma$ mediates protection by reducing HSV-2 infectivity to susceptible cells or increasing apoptosis of infected cells $(26,27)$. Inclusion of the latter mechanism in our model was crucial for recapitulating early viral load peak. The rapid lethality of diffusing cytokines explains the abrupt formation of clinical vesicles and erosions concurrent with viral clearance.

The generalized cytokine alarm state mechanism explains how HSV-2 elimination occurs rapidly within mucosal micro-regions defined by low HSV-specific $\mathrm{T}_{\mathrm{RM}}$ density (2). In our model, recognition of infected cells by a single pathogen-specific $T_{\mathrm{RM}}$ is necessary but insufficient for initiation of tissuewide protection. The timing of this sentinel event during the early hours of infection determines the extent of viral replication.

As was demonstrated in a mouse model, the total density of HSV-2 specific $\mathrm{T}_{\mathrm{RM}}$ is likely to be a key surrogate of immunity (24). At a scale of a $3 \mathrm{~mm}$ x $3 \mathrm{~mm}$ simulated micro-region, or a smaller 50cell region, $\mathrm{T}_{\mathrm{RM}}$ density maintains an inverse dose response with both peak viral load and shedding duration. Therefore, to achieve full protection, a therapeutic vaccine must achieve high HSV-2 specific $\mathrm{T}_{\mathrm{RM}}$ levels within all micro-regions across the genital region. During natural infection, the number of HSV-2 specific $\mathrm{T}_{\mathrm{RM}}$ is only sufficient to provide rapid protection in approximately $20 \%$ of tissue (20). The model projects that bystander $\mathrm{T}_{\mathrm{RM}}$, which cannot recognize infected cells, but secrete cytokines upon activation, play an important role in late elimination of infected cells during severe episodes. However, a high density of $\mathrm{T}_{\mathrm{RM}}$ that lack antigenic specificity is not predicted to be a surrogate of local immune protection. A high E:T ratio of HSV-2 specific cells is the key requirement for near sterilizing immunity.

Our in silico knock out analysis allows a detailed assessment of the relative contribution of $\mathrm{T}_{\mathrm{RM}}$ phenotypic characteristics to the pace of HSV-2 elimination. Single functions can typically be 
eliminated without dramatically impairing control of infection. For instance, elimination of trafficking or $\mathrm{T}_{\mathrm{RM}}$ dendricity has only modest effects on viral load during simulations; however, elimination of both functions leads to a substantial increase in episode duration and peak viral load. Cytokine effects also have substantial redundancy.

Our approach has important limitations pertaining to oversimplification of the totality of the immune response. We are unable to discriminate the effects of CD4+ and CD8+ $\mathrm{T}_{\mathrm{RM}},(28)$ and do not consider infiltrating natural killer cells (NK cells). CD8+ T cells co-localize with infected cells at the dermal-epidermal junction to a greater extent than $\mathrm{CD} 4+\mathrm{T}$ cells which provide help at a distance from the dermis (16). NK cells lack antigen specificity and are present in lower abundance in healing genital biopsy specimens $(8,16)$. Nevertheless, both cell populations are vital for control of human herpes viruses, exhibit memory, and secrete IFN- $\gamma$, perforin and granzyme B $(10,53)$. Therefore, NK cells and CD4 $+\mathrm{T}_{\mathrm{RM}}$ may serve as bystander cells and contribute to the general alarm state $(8)$. It will be important to distinguish the role of various infiltrating immune cell subsets in future models as relevant data accrues.

We do not include epithelial cell-induced innate immunity, antigen presenting cells (54), or antibody effect (55). While these mechanisms play a role in pathogen elimination, to our knowledge their effects may not be as variably dispersed over time and tissue space as $\mathrm{T}_{\mathrm{RM}}$. Therefore, our model may capture humoral and local innate immunity effects which are embedded within static model parameters for viral replication, infectivity and spread; if these effects vary in a non-linear fashion relative to local viral load, then our model misses these dynamics. During some contained episodes, study participants did not mount a robust IFN- $\alpha$ and IFN- $\beta$ response, indicating that epithelial-derived innate responses are sometimes suppressed. Yet, during severe episodes, it is possible that infected 
epithelial cells and antigen presenting cells sufficiently activate bystander $T_{\mathrm{RM}}$ even in the absence of HSV-specific $\mathrm{T}_{\mathrm{RM}}$.

We denote cytokines as one variable in our model, which is overly reductionist. The multiple antiviral effects of IFN- $\gamma$ are crucial, but a complex network of cytokines is likely to influence HSV-2 containment, with varying effects according to cellular source and cellular target. While a powerful cytokine impact on infected cells is a robust feature of our model, a more comprehensive assessment will only be possible as new data allows detailed parameterization of individual cytokine effects.

Our model projections deviate slightly from the observed data. The best fitting model does not achieve the highest peak viral loads, possibly because the two-dimensional model includes fewer target cells for HSV-2 replication than human skin. Our model also only simulates a single ulcer while swabbing protocols capture HSV-2 from multiple contemporaneous ulcers when viral loads are highest (19). The optimal model slightly overestimated the distribution of episode durations and peak viral loads at moderate ranks, perhaps because other infiltrating immune cell populations not included in the model contribute to control of infection.

Despite these limitations, modeling provides a unique platform to link observations from murine and human experiments, which is important because both of these approaches have inherent gaps. In humans, $\mathrm{T}_{\mathrm{RM}}$ responses evolve quickly within microscopic tissue environments and have not been captured during critical early stages of viral replication and spread. Successful containment of HSV-2 in a few cells, is asymptomatic, and even more difficult to observe empirically. Yet, quantitative components of human data, including heterogeneity in shedding episode kinetics and spatial density and topology of $\mathrm{T}_{\mathrm{RM}}$ serve as critical benchmarks for model validation.

Murine models utilizing intravital imaging allow observation and quantification of $\mathrm{T}_{\mathrm{RM}}$ behaviors. However, these experiments are rarely centered around realistic kinetics of pathogen control. 
Moreover, murine infection conditions may not be representative of human HSV-2 reactivation. It is plausible that $\mathrm{T}_{\mathrm{RM}}$ trafficking, dendricity, proliferation, synaptic killing and antiviral cytokine release occur in humans and mice. Yet, in vivo rates governing these behaviors may differ. Our analysis shows that knock out of many of these mechanisms results in perturbations in viral shedding outcomes.

HSV-2 infected individuals experience shedding episodes that are frequent and highly variable due to the spatial distribution of $\mathrm{T}_{\mathrm{RM}}$. We propose that containment of infection within low $\mathrm{T}_{\mathrm{RM}}$ density areas is contingent upon rapid diffusion of antiviral cytokines from activated $\mathrm{T}_{\mathrm{RM}}$. Effective vaccine strategies must increase the total number of $\mathrm{HSV}-2$ specific $\mathrm{T}_{\mathrm{RM}}$ across the entire genital tract and leverage the broad protection afforded by antiviral cytokines to induce a spatially homogeneous layer of immunological readiness. 


\section{Materials and Methods}

Study Design. We re-analyzed data from a previous study where HSV-2 seropositive, HIV-negative individuals were asked to self-collect genital swab specimens 4 times a day for 60 days. Viral load was measured using qPCR (30). For each participant, we divided the time series of viral loads into individual shedding episodes. We defined a single episode as a series of HSV-positive swabs with two negative swabs before and after. We discarded episodes where swabs were more than $12 \mathrm{~h}$ apart (i.e. 2 or more consecutive missing samples) and classified episodes as short (0 - $1 \mathrm{~d}]$, medium (1-2 d] and long ( $>2 \mathrm{~d})$. For each episode, we quantified peak viral load, viral load at the first positive swab, time to first episode peak and episode duration. Episode analysis and visualization were performed in $\mathrm{R}(56)$. We also analyzed viral swab data from 3 participants for both viral load and cytokine levels (described below).

Image analysis of human biopsy specimens. We re-analyzed RGB images from previously described human HSV-2 genital biopsy specimens showing cell nuclei (blue), CD4+ T cells (green) and CD8+ T cells (red) (20). Each of 10 participants was biopsied at 2 and 8 weeks after healing of the lesions. We included both biopsies in the analysis for each participant. We focused our analysis on CD8+ T cells in close proximity to the dermal-epidermal junction (DEJ), cropping all areas below the DEJ that were more than half the thickness of the epidermis below the dermal-epidermal junction. These cut areas typically had high neuronal density and low nuclear density. We enumerated CD8+ T cells and total nuclei representative of $\mathrm{T}$ cells and epithelial cells using the program Cell Profiler (57). Using a custom R script, we used the output from Cell Profiler for each image to analyze clustering of CD8+ T cells and to calculate E:T ratios within micro-regions from the biopsy. We started by dividing the image into micro-regions containing approximately 100 cells by performing k-means clustering on the positions of cell nuclei. We then assigned each CD8+ T cell to a micro-region by determining which cluster centroid 
was closest to each CD8+ T cell (Fig. 2b,c). Finally we computed E:T ratios for each cluster and for the biopsy overall. Code and data are available at http://github.com/proychou/SpatialHSV.

Cytokine data from swabs. Four study participants with recurrent HSV lesions were enrolled in an additional protocol in which swabs were obtained every 8 hours prior to lesion development. At the time of prodrome or ulcer formation, participants switched to every 3-hour sampling for 5 days. Two swabs were obtained at each timepoint. One was processed for HSV PCR (36), and the other was sent for MSD assay for cytokine quantitation (58).

\section{Individual-based model of viral spread and immunological containment of infection by $T_{R M}$. We}

developed a stochastic, agent-based, spatially explicit model of HSV infection that incorporates key features of host and virus life cycles and immune control by CD8 $+\mathrm{T}$ cells, based on a previously developed model of host-pathogen dynamics (59). The model is run on a 2D grid of sites; each site on the L x L grid is either vacant (no cell, no virus) or occupied by a single epithelial cell and one or more virus particles. In addition, each site may be patrolled by a CD8+ resident memory $\mathrm{T}$ cell $\left(\mathrm{T}_{\mathrm{RM}}\right)$ that is either HSV-specific or a bystander in the context of HSV infection. The size of the grid (L) ranged between $125 \times 125$ and $425 \times 425$ depending on the simulation.

At each site, we track the state of the epithelial cell, the state of any $\mathrm{T}_{\mathrm{RM}}$ patrolling the site, the number of infectious viruses present, and the concentration of cytokines at the site. At the start of the simulation, all sites are occupied by susceptible cells and a lawn of tissue-resident $\mathrm{T}$ cells at a predefined spatial density (defined as the probability that the focal site at the center of the grid contains $\mathrm{T}_{\mathrm{RM}}$ ). State transitions occur stochastically at probabilities that are determined by the rates of various events that can occur at the site described below (59). These rates determine the wait time, a random 
variable whose mean is the reciprocal of the corresponding rate. Virus and cytokine concentrations decay exponentially at rates specified in Tables 1-3. To avoid doubly propagating particles or adding dependencies on the order of cell evaluation, the change in values occurs across the grid at the end of each time step. Diffusion is described by a 2D-Gaussian function applied over a 3x3 neighborhood as described in (59).

For all simulations involving model fitting, we populate the grid with a spatial distribution of $\mathrm{T}_{\mathrm{RM}}$ drawn from the histologic slides from 10 participants in the human biopsy study described above (Fig. 2a). The E:T ratios from the image analysis above are used to generate rank order distributions of ratios for each biopsy and fit to an exponential curve of the form E:T ratio $=\mathrm{p}^{*} \mathrm{e}^{\mathrm{c}^{*} \text { rank }}$ (Fig. 2d). The resulting distributions of the coefficients $\mathrm{p}$ and $\mathrm{c}$ are used when seeding clusters of 100 cells in the simulated cell grid prior to each simulated episode.

Each susceptible cell at site (i, j) becomes infected at rate $\beta V_{i j}$, where $\beta$ is viral infectivity, and $\mathrm{V}_{\mathrm{ij}}$ is the number of viruses present at the site. Newly infected cells enter an eclipse phase during which they do not produce virus and then transition at rate $\varepsilon$ to a virus-producing state, producing $\mu$ free virus per unit time. Productively infected cells die at rate $\delta_{\mathrm{I}}$, while susceptible cells and pre-productively infected cells die at rate $\delta_{\mathrm{S}}$. Vacant sites and sites occupied by dead cells are repopulated by susceptible cells at rate $\alpha$. Each simulation starts with a single productively infected cell placed at the center of the grid and populations are recorded at 10-minute increments of simulated time for 5 days. Cell and virus parameters are listed in Table 1.

To this basic version of viral spread, we added immune mechanisms with features that can be switched on or off depending on the hypothesis being tested. When $\mathrm{T}_{\mathrm{RM}}$ are present, they patrol the grid at a specified rate. Movement can occur by either a "random walk", i.e. taking one step in a random direction within a 3 x 3 Moore neighborhood at a specified rate; "Levy walk", a modified random walk 
where step size is drawn from a Levy distribution; or "directed walk" where the T cell moves preferentially along a cytokine gradient (if present) and towards an infected cell when present within a 5 x 5 Moore neighborhood. For most simulations, unless specified, we assumed a random walk. T cell parameters are listed in Table 2.

When an HSV-specific $\mathrm{T}_{\mathrm{RM}}$ encounters an infected cell, movement stops and the $\mathrm{T}$ cell does one or more of the following: proliferates, kills the infected cell, and/or secretes cytokines. Rates of each of these processes were determined from the literature or inferred by fitting to data. The dendritic shape of $\mathrm{T}_{\mathrm{RM}}$ is modelled by allowing HSV-specific $\mathrm{T}_{\mathrm{RM}}$ to recognize and kill an infected cell within a 3 x 3 Moore neighborhood of its current position. In situ $\mathrm{T}$ cell proliferation is modeled by placing a daughter T cell at a randomly chosen vacant site within a 3 x 3 Moore neighborhood of the focal cell.

When activated, a T cell secretes cytokines at a defined rate and cytokines diffuse rapidly across the grid. When present at a site containing a keratinocyte, cytokines have multiple concentrationdependent effects including reducing infectivity of virus to susceptible cells, reducing viral replication rate in infected cells, and decreasing lifespan of infected cells. In addition, cytokines can activate bystander T cells that are not specific for HSV, allowing them to amplify the cytokine-mediated signal. Cytokine-related parameters are listed in Table 3. Cytokine effects (decreased viral replication, increased cell death rate, decreased viral infectivity and increased activation of surrounding $\mathrm{T}_{\mathrm{RM}}$ ) are modeled with the following equation:

$$
\text { effect }=\frac{1}{1+\frac{d o s e}{I C 50}}
$$

in which dose is the cytokine level (in $\mathrm{pg} / \mathrm{ml}$ ) within the single-cell region and IC50 is the level of cytokine level required to achieve half-maximal effect. The product is then multiplied by the parameter of relevance. 
Data fitting. We initially fit the model without $\mathrm{T}_{\mathrm{RM}}$ to achieve realistically high viral loads after 24 hours of shedding. Using a search algorithm and predefined ranges from the literature (Table 1), we tuned parameters of HSV-2 infectivity rate and diffusion rate, while fixing values for viral replication rate and clearance rate at experimentally observed values. We also tuned the model to diameter of the infected cell region from the empirical data. Simulated ulcer diameter was calculated from the number of dead cells $(18,60)$.

In order to evaluate models with and without various cytokine effects we used a fixed set of viral and $\mathrm{T}_{\mathrm{RM}}$ parameters and varied only the parameters associated with each of the four cytokine effects. Parameter sets that did not yield at least one large episode were discarded. A large episode was defined as generating the equivalent of a $1.5 \mathrm{~mm}$ diameter ulcer. We fit each model to the rank order distributions of the collected cohort data described above (Fig. 1) for episode peak viral load, time to peak and duration. The root sum of squares (RSS) was calculated by comparing the ranked values for the cohort data with ranked values for the simulated episodes. The squared error at each point was divided by the variance of the cohort measure in order to standardize the results across the different variables. The overall AIC score was calculated using the RSS score for each category together with a complexity variable $k$ that differed for each model based on the number of free parameters it introduced and a shared $n$ value that represented the number of data fitting points across the three rankings. The $n$ value was 83 unless the given parameter set yielded less than that number of episodes in 100 attempts. Each attempt started with one infected cell at the center of the grid. The equation used is shown below.

$$
A I C=n\left(\ln \frac{R S S_{\text {peak }}}{n}+\ln \frac{R S S_{\text {time to peak }}}{n}+\ln \frac{R S S_{\text {duration }}}{n}\right)+2 k
$$

The baseline model had no cytokine effects and was assigned a $k$ value of zero. The mean of the 10 highest AIC scores from parameter combinations for each of the 16 models was used to determine the best fit model (Fig. S2a). 
To further improve the fitting of the chosen model, we performed an additional round of simulations in which we varied viral diffusion, cytokine diffusion and cytokine uptake rates on a $\log 10$ scale across 500 parameter sets. All other parameters were held at the values for the best model. The final best parameter set from the initial fitting and fine-tuning exercise is shown in Table 3 .

In silico knock-out analyses. We ran simulations to evaluate the effect of removing single functions from the model and then measured results against the following metrics of episode severity: peak viral load, time to peak viral load, duration and total number of infected cells. Simulated sampling was every 6 hours to align with the cohort data in these model projections. Starting with the best parameter set for the full cytokine effects model, we ran additional simulations of 100 episodes each in which we removed one or more cytokine effects or $\mathrm{T}_{\mathrm{RM}}$ features. The cytokine effects removed were reduction in viral infectivity, viral production, acceleration of infected cell death and $\mathrm{T}_{\mathrm{RM}}$ activation. The latter was split into cytokine-modulated production of cytokine from bystander $\mathrm{T}_{\mathrm{RM}}$ and cytokine-modulated activation of both HSV-specific and bystander $\mathrm{T}_{\mathrm{RM}}$. The $\mathrm{T}_{\mathrm{RM}}$ effects included reducing dendricity, halting mobility and eliminating contact-mediated killing by HSV-specific $\mathrm{T}_{\mathrm{RM}}$. The resulting episodes were compared with those of the full model in several categories including average peak viral load, the total number of cells infected, episode duration and the percentage of episodes that were not controlled.

Code. All code and data are available at http://github.com/proychou/SpatialHSV. The model is available in both $\mathrm{R}$ and $\mathrm{C}++$ versions. 


\section{Supplementary Materials}

Fig. S1. Epithelial-derived antiviral cytokines do not predictably surge locally during HSV-2 shedding episodes.

Fig. S2. A model with all possible antiviral cytokine features optimizes fit to the data.

Fig. S3. Antiviral cytokines effects are polyfunctional and redundant within the HSV-2 infection microenvironment.

Fig. S4. Trafficking, dendricity, and contact-mediated killing, as well as activity of TRM accelerate but are not required for elimination of HSV-2 infected cells during severe episodes.

Movie S1. Lack of containment of HSV-2 spread in the absence of TRM.Movie S2. Lack of containment of HSV-2 spread in the presence of TRM which only kill via direct lysis of infected cells. Movie S3. Lack of containment of HSV-2 spread in the presence of TRM which only kill via direct lysis of infected cells.

Movie S4. Rapid containment of HSV-2 spread in the presence of TRM antiviral cytokine secretion and high TRM density.

Movie S5. Containment of HSV-2 spread in the presence of TRM antiviral cytokine secretion and low TRM density.

Movie S6. Containment of HSV-2 spread in the presence of TRM antiviral cytokine secretion and low TRM density.

Movie S7. Slower containment of HSV-2 spread in the presence of TRM antiviral cytokine secretion which only prevents infectivity.

Movie S8. Slightly protracted containment of HSV-2 spread in the presence of TRM antiviral cytokine secretion which only limit lifespan of infected cells. 
Table 1. Model parameters for cell and virus compartments in the absence of immunity

\begin{tabular}{|c|c|c|c|}
\hline Parameter & Unit & Value & Source \\
\hline Uninfected cell div $\operatorname{rate}^{1}(\alpha)$ & day $^{-1}$ & 0.077 & $(61)$ \\
\hline Uninfected cell death $\operatorname{rate}^{2}\left(\delta_{S}\right)$ & day $^{-1}$ & 0 & \\
\hline Infected cell death rate $\left(\delta_{\mathrm{I}}\right)$ & day $^{-1}$ & 1.25 & $(60)$ \\
\hline Lag in virus production ${ }^{3}(\varepsilon)$ & cell $^{-1}$ day $^{-1}$ & 8 & $(62)$ \\
\hline Infectivity $(\beta)$ & virus $^{-1}$ cell $^{-1}$ day $^{-1}$ & 0.1 & Fitted (initial model) \\
\hline Virus spread rate ${ }^{4}$ & sites day ${ }^{-1}$ & 54 & Fitted (final model) \\
\hline $\begin{array}{l}\text { Virus production rate } \\
(\mu, \text { mean } \pm \mathrm{SD})\end{array}$ & cell $^{-1}$ day $^{-1}$ & $3.16 \pm 1.00 \times 10^{4}$ & $(60)$ \\
\hline Free virus decay rate & site $^{-1}$ day $^{-1}$ & 8.8 & $(19)$ \\
\hline Spatial scale & $\mu \mathrm{m}$ site $^{-1}$ & 50 & $(63,64)$ \\
\hline
\end{tabular}

1 Rate at which a vacant site becomes occupied by a susceptible cell

2 We assumed that cell death due to infection greatly exceeds uninfected cell death during lesion formation.

3 This rate represents the time from infection to first released virus

4 Rate at which infectious virus spreads across the grid

Table 2. $\mathbf{T}_{\mathrm{RM}}$ parameters

\begin{tabular}{llll}
\hline Parameter & Unit & Value & Source \\
\hline $\mathrm{T}_{\text {RM }}$ starting density & - & varied & $(20)$ \\
HSV-specific fraction $^{1}$ & - & 0.4 & $(9)$ \\
$\mathrm{T}_{\mathrm{RM}}$ motility & sites day $^{-1}$ & 24 & $(1,22)$ \\
${\text { Death rate }(\mathrm{Ag}-)^{2}}_{\text {Proliferation }(\mathrm{Ag}+)}$ & day $^{-1}$ & 0 & $(65)$ \\
Killing rate $^{-1}$ & day $^{-1}$ & 3 & $(65,66)$ \\
Max T cell divisions & day $^{-1}$ & 48 & $(25)$ \\
\hline
\end{tabular}

1 Fraction of starting $\mathrm{T}_{\mathrm{RM}}$ that are HSV-specific, remaining are bystander $\mathrm{T}_{\mathrm{RM}}$

$2 \mathrm{~T}_{\mathrm{RM}}$ death rate in the absence of antigen

3 From antigen detection to death of infected cell, per CTL pairing

Table 3. Cytokine parameters

\begin{tabular}{|c|c|c|c|}
\hline Parameter & Unit & Value & Source \\
\hline Secretion by $T_{R M}$ & pg cell ${ }^{-1}$ day $^{-1}$ & 0.02 & (67) \\
\hline Diff & sites day ${ }^{-1}$ & 65 & Fitted \\
\hline Uptal & ${ }^{-1}$ day $^{-1}$ & 6.9 & Fitted \\
\hline Decay rate & site $^{-1}$ day $^{-1}$ & 0.01 & Fitted \\
\hline IC50: infected c & pg cell- ${ }^{-1}$ & $5.4 \times 10^{-6}$ & Fitted \\
\hline nfectivity & $\mathrm{pg} \mathrm{c}$ & $7.0 \times 10^{-7}$ & Fitted \\
\hline IC50: & $\mathrm{pg} \mathrm{c}$ & $1.0 \times 10^{-6}$ & Fitted \\
\hline IC50: $T_{R M}$ activation & pg cell ${ }^{-1}$ & $1.6 \times 10^{-7}$ & Fitted \\
\hline
\end{tabular}




\section{References}

1. S. Ariotti, J. B. Beltman, G. Chodaczek, M. E. Hoekstra, A. E. van Beek, R. Gomez-Eerland, L. Ritsma, J. van Rheenen, A. F. Maree, T. Zal, R. J. de Boer, J. B. Haanen, T. N. Schumacher, Tissue-resident memory CD8 + T cells continuously patrol skin epithelia to quickly recognize local antigen. Proc Natl Acad Sci U S A 109, 19739-19744 (2012).

2. S. Ariotti, M. A. Hogenbirk, F. E. Dijkgraaf, L. L. Visser, M. E. Hoekstra, J. Y. Song, H. Jacobs, J. B. Haanen, T. N. Schumacher, T cell memory. Skin-resident memory CD8(+) T cells trigger a state of tissue-wide pathogen alert. Science 346, 101-105 (2014).

3. T. Gebhardt, U. Palendira, D. C. Tscharke, S. Bedoui, Tissue-resident memory T cells in tissue homeostasis, persistent infection, and cancer surveillance. Immunol Rev 283, 54-76 (2018).

4. T. Gebhardt, L. M. Wakim, L. Eidsmo, P. C. Reading, W. R. Heath, F. R. Carbone, Memory T cells in nonlymphoid tissue that provide enhanced local immunity during infection with herpes simplex virus. Nat Immunol 10, 524-530 (2009).

5. L. K. Mackay, A. Rahimpour, J. Z. Ma, N. Collins, A. T. Stock, M. L. Hafon, J. Vega-Ramos, P. Lauzurica, S. N. Mueller, T. Stefanovic, D. C. Tscharke, W. R. Heath, M. Inouye, F. R. Carbone, T. Gebhardt, The developmental pathway for CD103(+)CD8+ tissue-resident memory T cells of skin. Nat Immunol 14, 1294-1301 (2013).

6. L. K. Mackay, A. T. Stock, J. Z. Ma, C. M. Jones, S. J. Kent, S. N. Mueller, W. R. Heath, F. R. Carbone, T. Gebhardt, Long-lived epithelial immunity by tissue-resident memory T (TRM) cells in the absence of persisting local antigen presentation. Proc Natl Acad Sci U S A 109, 7037-7042 (2012).

7. D. Masopust, V. Vezys, A. L. Marzo, L. Lefrancois, Preferential localization of effector memory cells in nonlymphoid tissue. Science 291, 2413-2417 (2001). 
8. J. M. Schenkel, K. A. Fraser, L. K. Beura, K. E. Pauken, V. Vezys, D. Masopust, T cell memory. Resident memory CD8 T cells trigger protective innate and adaptive immune responses. Science 346, 98-101 (2014).

9. J. Zhu, D. Koelle, J. Cao, J. Vazquez, M. Huang, F. Hladik, A. Wald, L. Corey, Virus-specific CD8+ T cells accumulate near sensory nerve endings in genital skin during subclinical HSV-2 reactivation. $J$ Exp Med 204, 595-603 (2007).

10. J. Zhu, T. Peng, C. Johnston, K. Phasouk, A. S. Kask, A. Klock, L. Jin, K. Diem, D. M. Koelle, A. Wald, H. Robins, L. Corey, Immune surveillance by CD8alphaalpha+ skin-resident T cells in human herpes virus infection. Nature 497, 494-497 (2013).

11. H. Shin, A. Iwasaki, A vaccine strategy that protects against genital herpes by establishing local memory T cells. Nature 491, 463-467 (2012).

12. L. K. Mackay, M. Minnich, N. A. Kragten, Y. Liao, B. Nota, C. Seillet, A. Zaid, K. Man, S. Preston, D. Freestone, A. Braun, E. Wynne-Jones, F. M. Behr, R. Stark, D. G. Pellicci, D. I. Godfrey, G. T. Belz, M. Pellegrini, T. Gebhardt, M. Busslinger, W. Shi, F. R. Carbone, R. A. van Lier, A. Kallies, K. P. van Gisbergen, Hobit and Blimp1 instruct a universal transcriptional program of tissue residency in lymphocytes. Science 352, 459-463 (2016).

13. B. V. Kumar, W. Ma, M. Miron, T. Granot, R. S. Guyer, D. J. Carpenter, T. Senda, X. Sun, S. H. Ho, H. Lerner, A. L. Friedman, Y. Shen, D. L. Farber, Human Tissue-Resident Memory T Cells Are Defined by Core Transcriptional and Functional Signatures in Lymphoid and Mucosal Sites. Cell Rep 20, 2921-2934 (2017).

14. T. Sathaliyawala, M. Kubota, N. Yudanin, D. Turner, P. Camp, J. J. Thome, K. L. Bickham, H. Lerner, M. Goldstein, M. Sykes, T. Kato, D. L. Farber, Distribution and compartmentalization of human circulating and tissue-resident memory T cell subsets. Immunity 38, 187-197 (2013). 
15. J. J. Milner, C. Toma, B. Yu, K. Zhang, K. Omilusik, A. T. Phan, D. Wang, A. J. Getzler, T. Nguyen, S. Crotty, W. Wang, M. E. Pipkin, A. W. Goldrath, Runx3 programs CD8(+) T cell residency in non-lymphoid tissues and tumours. Nature 552, 253-257 (2017).

16. J. Zhu, F. Hladik, A. Woodward, A. Klock, T. Peng, C. Johnston, M. Remington, A. Magaret, D. M. Koelle, A. Wald, L. Corey, Persistence of HIV-1 receptor-positive cells after HSV-2 reactivation is a potential mechanism for increased HIV-1 acquisition. Nat Med 15, 886-892 (2009).

17. J. T. Schiffer, Mucosal HSV-2 Specific CD8+ T-Cells Represent Containment of Prior Viral Shedding Rather than a Correlate of Future Protection. Front Immunol 4, 209 (2013).

18. J. T. Schiffer, L. Abu-Raddad, K. E. Mark, J. Zhu, S. Selke, D. M. Koelle, A. Wald, L. Corey, Mucosal host immune response predicts the severity and duration of herpes simplex virus-2 genital tract shedding episodes. Proc Natl Acad Sci U S A 107, 18973-18978 (2010).

19. J. T. Schiffer, D. Swan, R. Al Sallaq, A. Magaret, C. Johnston, K. E. Mark, S. Selke, N. Ocbamichael, S. Kuntz, J. Zhu, B. Robinson, M. L. Huang, K. R. Jerome, A. Wald, L. Corey, Rapid localized spread and immunologic containment define Herpes simplex virus-2 reactivation in the human genital tract. Elife 2, e00288 (2013).

20. J. T. Schiffer, D. A. Swan, P. Roychoudhury, J. M. Lund, M. Prlic, J. Zhu, A. Wald, L. Corey, A Fixed Spatial Structure of CD8(+) T Cells in Tissue during Chronic HSV-2 Infection. J Immunol 201, 1522-1535 (2018).

21. J. T. Schiffer, A. Wald, S. Selke, L. Corey, A. Magaret, The kinetics of mucosal herpes simplex virus-2 infection in humans: evidence for rapid viral-host interactions. J Infect Dis 204, 554-561 (2011). 
22. A. Zaid, L. K. Mackay, A. Rahimpour, A. Braun, M. Veldhoen, F. R. Carbone, J. H. Manton, W. R. Heath, S. N. Mueller, Persistence of skin-resident memory T cells within an epidermal niche. Proc Natl Acad Sci U S A 111, 5307-5312 (2014).

23. L. K. Beura, J. S. Mitchell, E. A. Thompson, J. M. Schenkel, J. Mohammed, S. Wijeyesinghe, R. Fonseca, B. J. Burbach, H. D. Hickman, V. Vezys, B. T. Fife, D. Masopust, Intravital mucosal imaging of $\mathrm{CD} 8(+)$ resident memory $\mathrm{T}$ cells shows tissue-autonomous recall responses that amplify secondary memory. Nat Immunol 19, 173-182 (2018).

24. S. L. Park, A. Zaid, J. L. Hor, S. N. Christo, J. E. Prier, B. Davies, Y. O. Alexandre, J. L. Gregory, T. A. Russell, T. Gebhardt, F. R. Carbone, D. C. Tscharke, W. R. Heath, S. N. Mueller, L. K. Mackay, Local proliferation maintains a stable pool of tissue-resident memory T cells after antiviral recall responses. Nat Immunol 19, 183-191 (2018).

25. S. Halle, K. A. Keyser, F. R. Stahl, A. Busche, A. Marquardt, X. Zheng, M. Galla, V. Heissmeyer, K. Heller, J. Boelter, K. Wagner, Y. Bischoff, R. Martens, A. Braun, K. Werth, A. Uvarovskii, H. Kempf, M. Meyer-Hermann, R. Arens, M. Kremer, G. Sutter, M. Messerle, R. Forster, In Vivo Killing Capacity of Cytotoxic T Cells Is Limited and Involves Dynamic Interactions and T Cell Cooperativity. Immunity 44, 233-245 (2016).

26. M. E. Dobbs, J. E. Strasser, C. F. Chu, C. Chalk, G. N. Milligan, Clearance of herpes simplex virus type 2 by CD8+ T cells requires gamma interferon and either perforin- or Fas-mediated cytolytic mechanisms. J Virol 79, 14546-14554 (2005).

27. T. Peng, J. Zhu, Y. Hwangbo, L. Corey, R. E. Bumgarner, Independent and cooperative antiviral actions of beta interferon and gamma interferon against herpes simplex virus replication in primary human fibroblasts. J Virol 82, 1934-1945 (2008). 
28. N. Iijima, A. Iwasaki, T cell memory. A local macrophage chemokine network sustains protective tissue-resident memory CD4 T cells. Science 346, 93-98 (2014).

29. K. Thurley, D. Gerecht, E. Friedmann, T. Hofer, Three-Dimensional Gradients of Cytokine Signaling between T Cells. PLoS Comput Biol 11, e1004206 (2015).

30. K. E. Mark, A. Wald, A. S. Magaret, S. Selke, L. Olin, M. L. Huang, L. Corey, Rapidly cleared episodes of herpes simplex virus reactivation in immunocompetent adults. J Infect Dis 198, 1141-1149 (2008).

31. L. Corey, K. K. Holmes, Genital herpes simplex virus infections: current concepts in diagnosis, therapy, and prevention. Ann Intern Med 98, 973-983 (1983).

32. K. E. Mark, A. Wald, A. S. Magaret, S. Selke, S. Kuntz, M. L. Huang, L. Corey, Rapidly cleared episodes of oral and anogenital herpes simplex virus shedding in HIV-infected adults. $J$ Acquir Immune Defic Syndr 54, 482-488 (2010).

33. J. T. Schiffer, A. Magaret, S. Selke, L. Corey, A. Wald, Detailed analysis of mucosal herpes simplex virus-2 replication kinetics with and without antiviral therapy. J Antimicrob Chemother 66, 2593-2600 (2011).

34. J. T. Schiffer, D. A. Swan, A. Magaret, L. Corey, A. Wald, J. Ossig, H. Ruebsamen-Schaeff, S. Stoelben, B. Timmler, H. Zimmermann, M. R. Melhem, S. A. Van Wart, C. M. Rubino, A. Birkmann, Mathematical modeling of herpes simplex virus-2 suppression with pritelivir predicts trial outcomes. Sci Transl Med 8, 324ra315 (2016).

35. A. Wald, J. Zeh, S. Selke, T. Warren, R. Ashley, L. Corey, Genital Shedding of Herpes Simplex Virus among Men. J Infect Dis 186 Suppl 1, S34-39 (2002). 
36. A. Wald, J. Zeh, S. Selke, T. Warren, A. Ryncarz, R. Ashley, J. Krieger, L. Corey, Reactivation of genital herpes simplex virus type 2 infection in asymptomatic seropositive persons. $N$ Engl $J$ Med 342, 844-850 (2000).

37. C. Johnston, J. Zhu, L. Jing, K. J. Laing, C. M. McClurkan, A. Klock, K. Diem, L. Jin, J. Stanaway, E. Tronstein, W. W. Kwok, M. L. Huang, S. Selke, Y. Fong, A. Magaret, D. M. Koelle, A. Wald, L. Corey, Virologic and immunologic evidence of multifocal genital herpes simplex virus 2 infection. $J$ Virol 88, 4921-4931 (2014).

38. K. D. Roizman B, Whitley RJ. The replication of Herpes simplex viruses. In: Knipe DM, et al., editors. Fields' Virology. 5th Ed. New York: Lippincott-Williams and Wilkins; 2007. pp. 25012601.

39. B. Galen, N. Cheshenko, A. Tuyama, B. Ramratnam, B. C. Herold, Access to nectin favors herpes simplex virus infection at the apical surface of polarized human epithelial cells. $J$ Virol 80, 12209-12218 (2006).

40. J. C. Carmichael, H. Yokota, R. C. Craven, A. Schmitt, J. W. Wills, The HSV-1 mechanisms of cell-to-cell spread and fusion are critically dependent on host PTP1B. PLoS Pathog 14, e1007054 (2018)

41. A. L. Bauer, C. A. Beauchemin, A. S. Perelson, Agent-based modeling of host-pathogen systems: The successes and challenges. Inf Sci (N Y) 179, 1379-1389 (2009).

42. J. T. Schiffer, D. A. Swan, A. Magaret, T. W. Schacker, A. Wald, L. Corey, Mathematical Modeling Predicts that Increased HSV-2 Shedding in HIV-1 Infected Persons Is Due to Poor Immunologic Control in Ganglia and Genital Mucosa. PLoS One 11, e0155124 (2016). 
43. G. M. Fricke, K. A. Letendre, M. E. Moses, J. L. Cannon, Persistence and Adaptation in Immunity: T Cells Balance the Extent and Thoroughness of Search. PLoS Comput Biol 12, e1004818 (2016)

44. P. J. Choi, T. J. Mitchison, Imaging burst kinetics and spatial coordination during serial killing by single natural killer cells. Proc Natl Acad Sci U S A 110, 6488-6493 (2013).

45. J. K. Whitmire, J. T. Tan, J. L. Whitton, Interferon-gamma acts directly on CD8+ T cells to increase their abundance during virus infection. $J$ Exp Med 201, 1053-1059 (2005).

46. J. M. Curtsinger, P. Agarwal, D. C. Lins, M. F. Mescher, Autocrine IFN-gamma promotes naive CD8 $\mathrm{T}$ cell differentiation and synergizes with IFN-alpha to stimulate strong function. J Immunol 189, 659-668 (2012).

47. K. Tewari, Y. Nakayama, M. Suresh, Role of direct effects of IFN-gamma on T cells in the regulation of CD8 T cell homeostasis. J Immunol 179, 2115-2125 (2007).

48. P. Bhat, G. Leggatt, N. Waterhouse, I. H. Frazer, Interferon-gamma derived from cytotoxic lymphocytes directly enhances their motility and cytotoxicity. Cell Death Dis 8, e2836 (2017).

49. T. Chu, A. J. Tyznik, S. Roepke, A. M. Berkley, A. Woodward-Davis, L. Pattacini, M. J. Bevan, D. Zehn, M. Prlic, Bystander-activated memory CD8 T cells control early pathogen load in an innate-like, NKG2D-dependent manner. Cell Rep 3, 701-708 (2013).

50. J. T. Schiffer, D. A. Swan, M. Prlic, J. M. Lund, Herpes simplex virus-2 dynamics as a probe to measure the extremely rapid and spatially localized tissue-resident T-cell response. Immunol Rev 285, $113-133$ (2018).

51. J. T. Schiffer, B. T. Mayer, Y. Fong, D. A. Swan, A. Wald, Herpes simplex virus-2 transmission probability estimates based on quantity of viral shedding. J R Soc Interface 11, 20140160 (2014). 
52. L. Corey, A. Wald, R. Patel, S. Sacks, S. Tyring, T. Warren, J. J. Douglas, J. Paavonen, R. Morrow, K. Beutner, L. Stratchounsky, G. Mertz, O. Keene, H. Watson, D. Tait, M. VargasCortes, Once-daily valacyclovir to reduce the risk of transmission of genital herpes. $N$ Engl $J$ Med 350, 11-20 (2004).

53. T. Peng, J. Zhu, K. Phasouk, D. M. Koelle, A. Wald, L. Corey, An effector phenotype of CD8+ T cells at the junction epithelium during clinical quiescence of herpes simplex virus 2 infection. $J$ Virol 86, 10587-10596 (2012).

54. M. Kim, N. R. Truong, V. James, L. Bosnjak, K. J. Sandgren, A. N. Harman, N. Nasr, K. M. Bertram, N. Olbourne, S. Sawleshwarkar, K. McKinnon, R. C. Cohen, A. L. Cunningham, Relay of herpes simplex virus between Langerhans cells and dermal dendritic cells in human skin. PLoS Pathog 11, e1004812 (2015).

55. C. Petro, P. A. Gonzalez, N. Cheshenko, T. Jandl, N. Khajoueinejad, A. Benard, M. Sengupta, B. C. Herold, W. R. Jacobs, Herpes simplex type 2 virus deleted in glycoprotein D protects against vaginal, skin and neural disease. Elife 4, (2015).

56. R. C. Team, R: A Language and Environment for Statistical Computing. https://www.Rproject.org. 2015.

57. A. E. Carpenter, T. R. Jones, M. R. Lamprecht, C. Clarke, I. H. Kang, O. Friman, D. A. Guertin, J. H. Chang, R. A. Lindquist, J. Moffat, P. Golland, D. M. Sabatini, CellProfiler: image analysis software for identifying and quantifying cell phenotypes. Genome Biol 7, R100 (2006).

58. E. Staples, R. J. Ingram, J. C. Atherton, K. Robinson, Optimising the quantification of cytokines present at low concentrations in small human mucosal tissue samples using Luminex assays. $J$ Immunol Methods 394, 1-9 (2013). 
59. P. Roychoudhury, N. Shrestha, V. R. Wiss, S. M. Krone, Fitness benefits of low infectivity in a spatially structured population of bacteriophages. Proc Biol Sci 281, 20132563 (2014).

60. J. T. Schiffer, L. Abu-Raddad, K. E. Mark, J. Zhu, S. Selke, A. Magaret, A. Wald, L. Corey, Frequent release of low amounts of herpes simplex virus from neurons: results of a mathematical model. Sci Transl Med 1, 7ra16 (2009).

61. G. D. Weinstein, J. L. McCullough, P. Ross, Cell proliferation in normal epidermis. J Invest Dermatol 82, 623-628 (1984).

62. J. D. Smith, E. De Harven, Herpes simplex virus and human cytomegalovirus replication in WI38 cells. I. Sequence of viral replication. J Virol 12, 919-930 (1973).

63. M. E. S. Brunzel NA. Fundamentals of urine \& body fluid analysis. 3rd ed. St. Louis.

64. H. Evers, C. G. Birngruber, F. Ramsthaler, U. Muller, S. Bruck, M. A. Verhoff, [Differentiation of epithelial cell types by cell diameter]. Arch Kriminol 228, 11-19 (2011).

65. R. J. De Boer, M. Oprea, R. Antia, K. Murali-Krishna, R. Ahmed, A. S. Perelson, Recruitment times, proliferation, and apoptosis rates during the $\mathrm{CD} 8(+) \mathrm{T}$-cell response to lymphocytic choriomeningitis virus. J Virol 75, 10663-10669 (2001).

66. M. Lenardo, K. M. Chan, F. Hornung, H. McFarland, R. Siegel, J. Wang, L. Zheng, Mature T lymphocyte apoptosis--immune regulation in a dynamic and unpredictable antigenic environment. Annu Rev Immunol 17, 221-253 (1999).

67. J. E. Wigginton, D. Kirschner, A model to predict cell-mediated immune regulatory mechanisms during human infection with Mycobacterium tuberculosis. J Immunol 166, 1951-1967 (2001). 
Acknowledgements: We express gratitude to our dedicated study participants, clinical research staff (Nui Pholsena, Dana Varon and Jessica Moreno), molecular laboratory scientists (Meei-Li Huang) and to helpful scientific input from Daniel Reeves, Florencia Tettamanti Boshier, and Fabian Cardozo Ojeda.

Funding: This work was supported by National Institute of Allergy and Infectious Diseases Grant P01 AI030731 to J.T.S. and Grant R01 AI121129 to J.T.S., J.M.L., and M.P.

Author contributions: P.R. conceived the study, performed mathematical modeling, performed statistical analyses, and wrote the manuscript. D.A.S. performed spatial analyses of biopsy specimens, mathematical modeling and statistical analyses. E.D. assisted with literature review and model parameter selection. L.C. conceived the biopsy study. J.Z. performed staining of biopsy specimens. V.D. and L.R.S performed cytokine analysis. J.M.L. and M.P. conceived the study and edited the manuscript. J.T.S. conceived the study, performed mathematical modeling, performed statistical analyses, and wrote the manuscript.

Competing interests: L.C. is on the scientific advisory board for and holds stock $(<1 \%$ of the company) in Immune Design Corp. and is a coinventor listed on several patents involving potential HSV vaccine development. J.T.S. received research funds from Genocea. The other authors have no financial conflicts of interest.

Data and materials availability: All code and data are available at http://github.com/proychou/SpatialHSV. 


\section{Figures:}
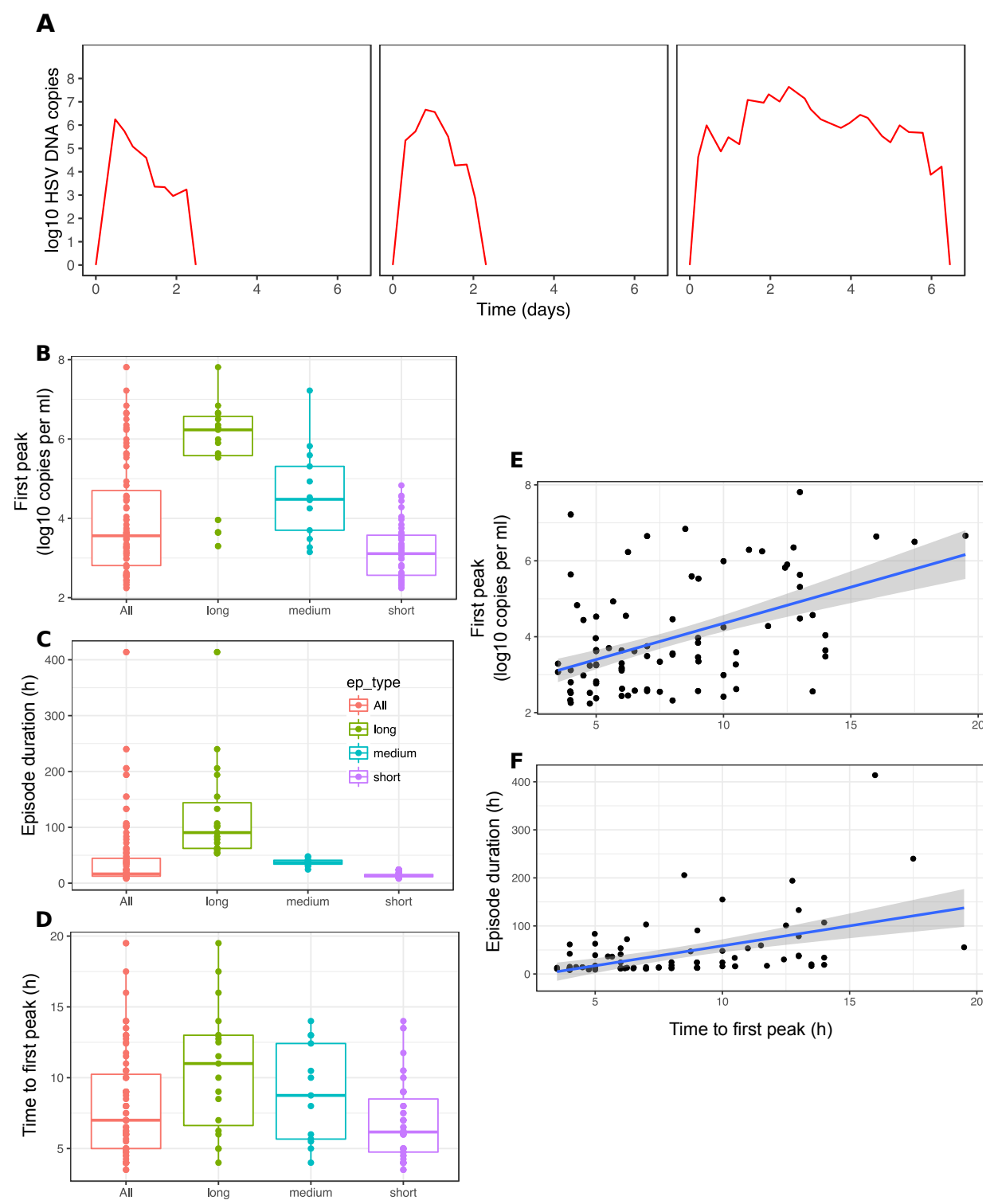

Fig. 1. Early and intense immunological pressure against HSV-2 replication within genital micro-environments. (A) Three severe shedding episodes with typical characteristics including rapid time to first peak viral load and rapid viral clearance. (B-D) Analysis of 83 shedding episodes derived from every 6-hour genital sampling. Boxplots with interquartile range or IQR (box) and 1.5x the IQR (whiskers). Categorization according to duration: short $<24$ hours $(n=51)$, medium $24-48$ hours $(n=13)$, and long $>48$ hours $(n=19)$. (B) Variable peak viral loads and (C) durations across episodes with most episodes eliminated in fewer than 48 hours. (D) Uniform occurrence of first peak viral load within the first 24 hours of HSV-2 detection. (E) Correlation of time to peak viral load with peak episode viral load (Spearman's $\rho=0.434$, $\mathrm{p}<0.001$ ). (F) Correlation of time to peak viral load with episode duration (Spearman's $\rho=0.534$, $\mathrm{p}<0.001)$. 

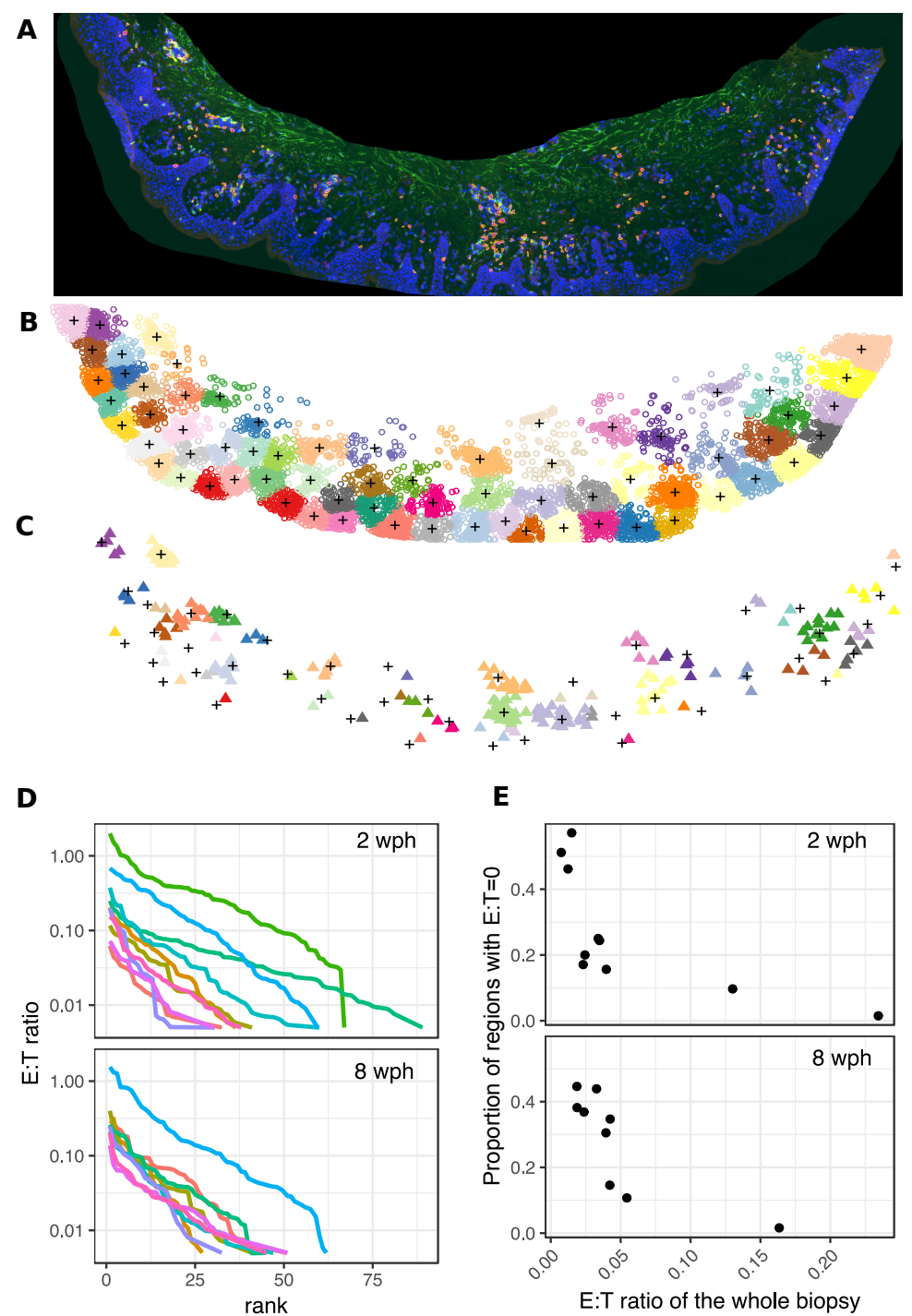

Fig. 2. Heterogeneous dispersion of CD8+ T cells within HSV-2 infected genital biopsy specimens obtained after lesion healing. Analysis of 19 genital biopsy specimens from 10 participants 2 weeks $(n=10)$ or 8 weeks $(n=9)$ after lesion healing. (A) Three-color RGB image with cell nuclei (blue), CD4+ T cells (green), and CD8+ T cells (red) of a 2-week specimen. (B) 100-epidermal cell micro-regions designated according to clusters shown as separate colors; "+" $=$ cluster center. (C) CD8+ T cells (triangles) within each micro-region. (D) Rank order distributions of CD8+ T cell : epithelial (E:T) ratios within micro-regions; lines = single specimens; wide ranges of E:T ratios within each specimen with similar rank order slopes across specimens. (E) High variability of whole specimen E:T ratios (x-axis) across 2- and 8-week specimens; inverse correlation of whole specimen E:T ratios with the proportion of 100 keratinocyte regions containing no CD8+ T cells (y-axis). 


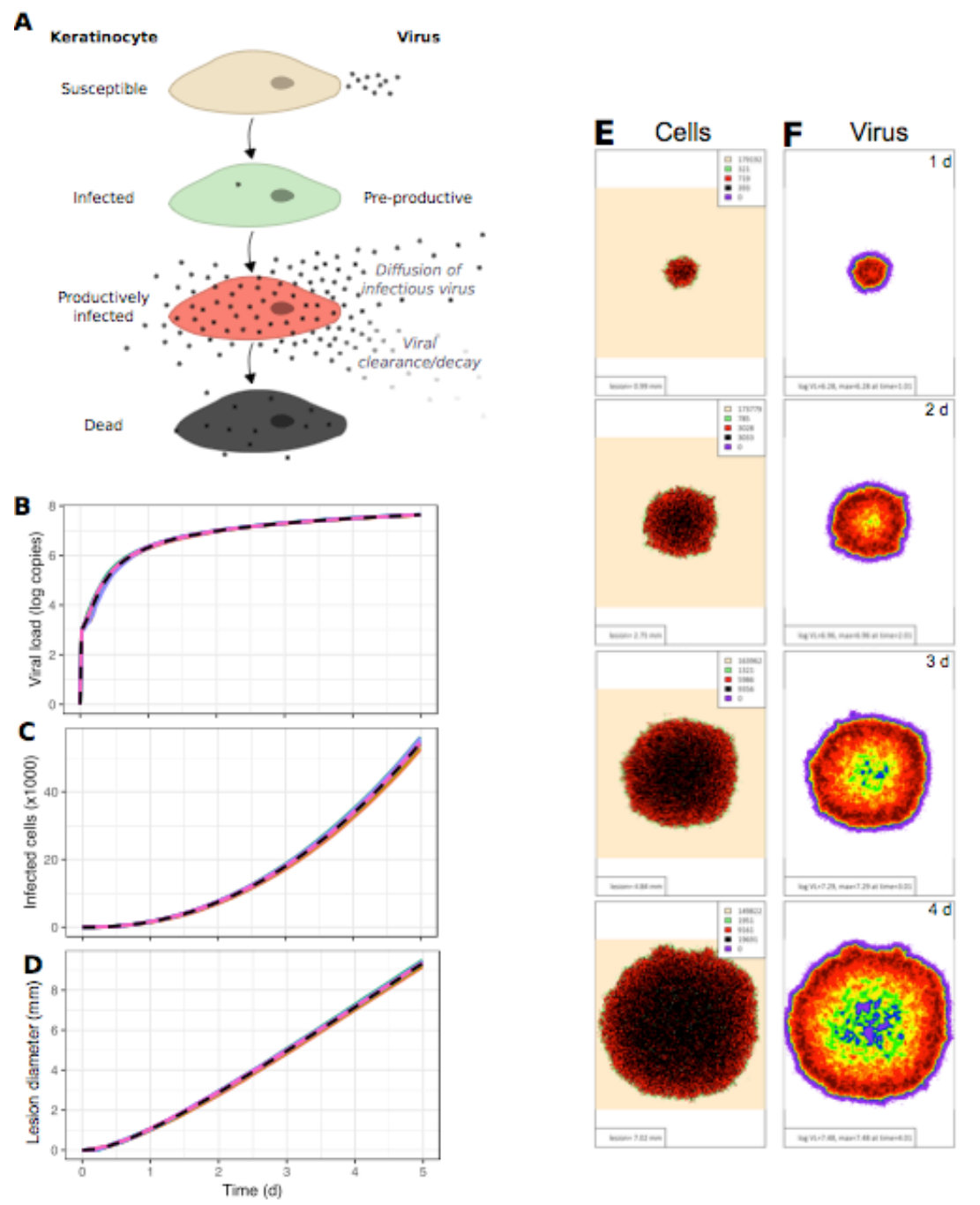

Fig. 3. Continual spatial expansion of HSV-2 infection in mathematical model simulations without $\mathbf{T}_{\text {RM. }}$ (A) Mathematical model of HSV-2 replication and spread; infected cell progression through pre-productive (green), and productive phases (red) during which viral replication occurs and virus diffuses to surrounding regions. (B) HSV-2 viral load trajectory versus time for 10 simulated episodes with asymptotic behavior and no viral elimination (dotted line $=$ median). (C) Number of infected cells and (D) ulcer diameter versus time with continual expansion in the absence of a $\mathrm{T}_{\mathrm{RM}}$ response in the 10 simulated episodes. (E-F) Spatial approximation of infection spread in 5-day simulations which correspond to Movie S1; one-day time steps per row. (E) An expanding ulcer with a leading edge of pre-productively infected cells (green), an inner ring of productively infected cells (red) and a core of dead cells (black). (F) Viral diffusion extending beyond the range of infected cells with highest local viral loads noted over regions with active HSV-2 replication; viral loads (HSV DNA copies) per cellular region: purple $=10^{2}-10^{2.25}$, blue $=10^{2.25}-10^{2.5}$, green $=10^{2.5}-10^{2.75}$, yellow $=10^{2.75}-10^{3}$, orange $=10^{3}-10^{3.25}$, red $=10^{3.25}-10^{3.5}$, dark red $>10^{3.5}$. 

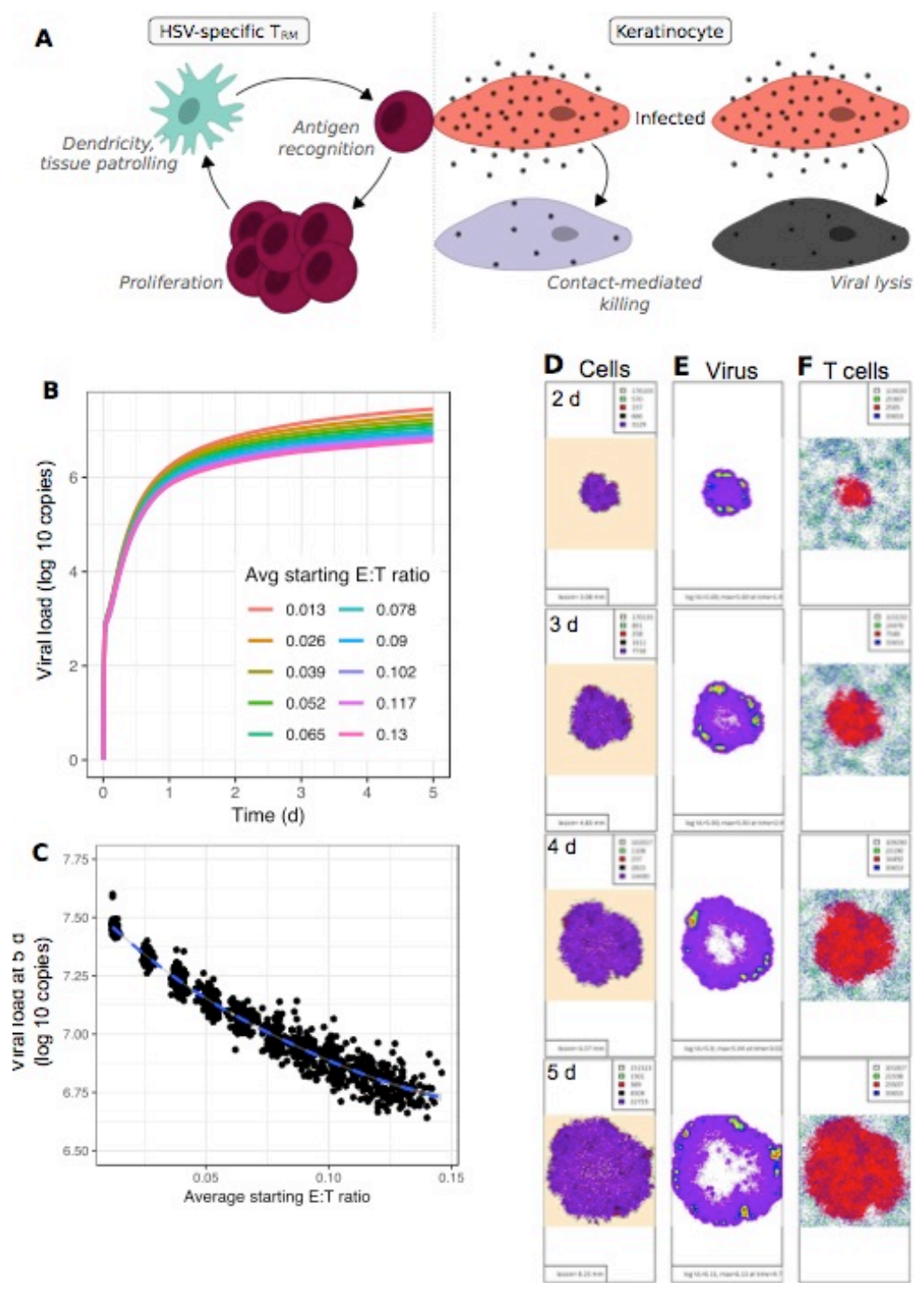

Fig. 4. Failed elimination of infected cells at low $\mathrm{E}: \mathrm{T}$ ratios in mathematical model simulations in which $\mathbf{T}_{\mathbf{R}}$ only exert contact mediated killing. (A) Model schematic: between HSV-2 shedding episodes, $\mathrm{T}_{\mathrm{RM}}$ immunosurveillance by patrolling and expression of dendritic arms; during episodes, local $\mathrm{T}_{\mathrm{RM}}$ proliferation and killing of adjacent infected cells. (B-C) Simulated episodes at different E:T ratios. (B) Viral load trajectories with asymptotic behavior and lower setpoints at higher starting E:T ratios. (C) Inverse correlation between E:T ratio and viral load at day 5 (dot $=$ a simulation, $\mathrm{n}=1000)$. (D-F) Spatial infection at high E:T ratios in 5day simulations which correspond to Movie S2; one-day time steps per row. (D) Preproductively infected cells (green) at the leading edge with a limited inner ring of productively infected cells (red), a core of virally lysed cells (black) and mostly $\mathrm{T}_{\mathrm{RM}}$ lysed cells (purple cells). (E) Active HSV-2 replication within isolated intense foci of high local viral loads over regions with actively infected cells; viral loads (HSV DNA copies) per cellular region: purple $=10^{2}-10^{2.25}$, blue $=10^{2.25}-10^{2.5}$, green $=10^{2.5}-10^{2.75}$, yellow $=10^{2.75}-10^{3}$, orange $=10^{3}-10^{3.25}$, red $=10^{3.25}-10^{3.5}$, dark red $>10^{3.5}$. (F) HSV-2 specific $\mathrm{T}_{\mathrm{RM}}$ expansion during lesion spread (green $=$ inactivated $\mathrm{HSV}$-specific $\mathrm{T}_{\mathrm{RM}}$, red = activated $\mathrm{HSV}$-specific $\mathrm{T}_{\mathrm{RM}}$, blue= inactivated bystander $\left.\mathrm{T}_{\mathrm{RM}}\right)$. 

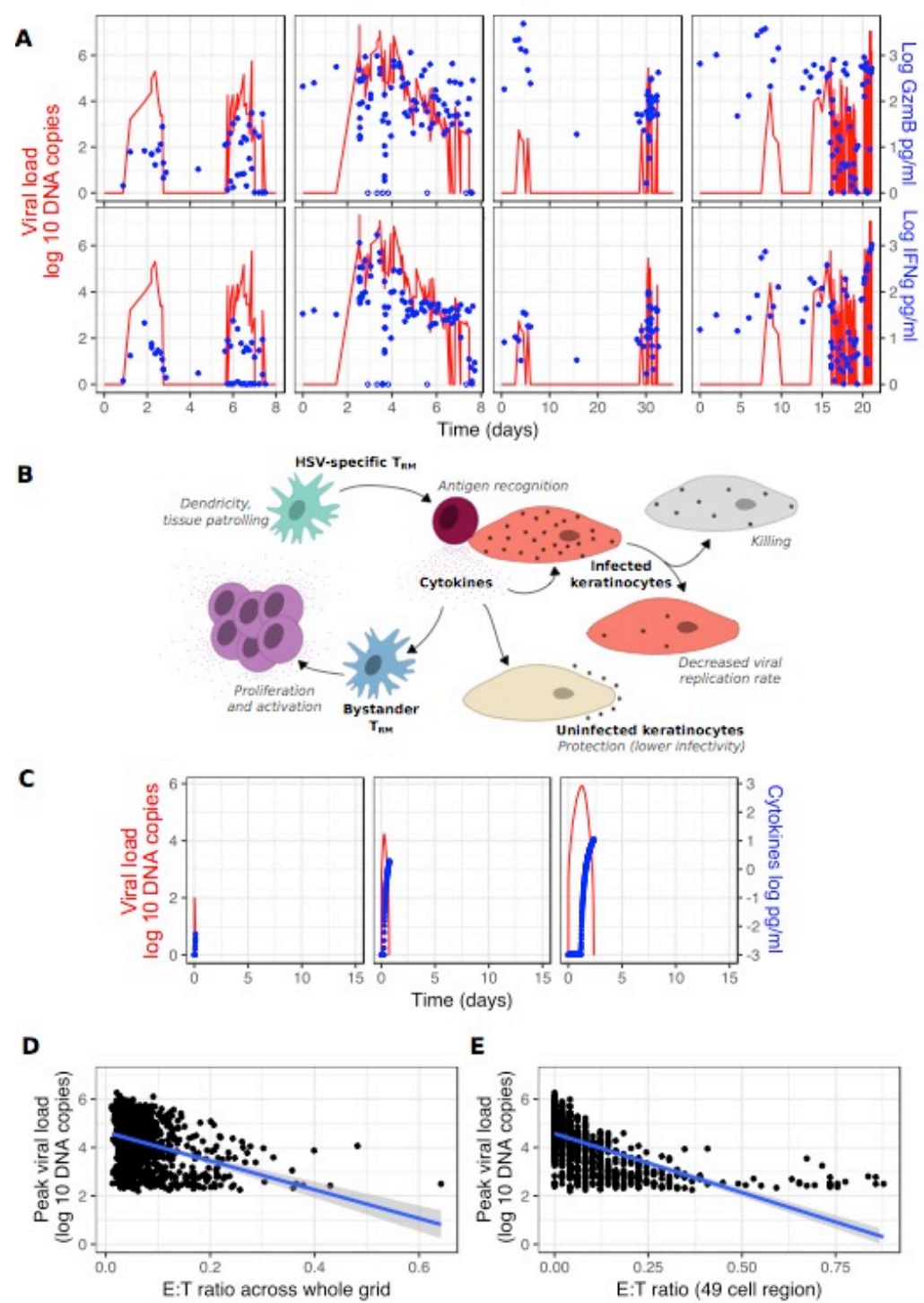

Fig. 5. $T_{\text {RM }}$ induced cytokine surge leading to rapid elimination of infected cells. (A) HSV-2 shedding episodes in four infected persons who swabbed every 8 hours pre-lesion detection and every 3 hours post-lesion detection; equivalent episodes aligned vertically; red line = HSV-2 DNA (left y-axis). (A, top row); surge in granzyme B (blue dots) during viral expansion indicating T-cell mediated killing directly via the T cell receptor. (A, bottom row); surge in IFN- $\gamma$ levels (blue dots) indicating secretion and paracrine effects by local $\mathrm{T}_{\mathrm{RM}}$. (B) Mathematical model schematic: possible cytokine effects including enhanced killing of infected cells, lowering viral replication rate in infected cells, lowering HSV-2 infectivity to uninfected cells and activating other bystander $\mathrm{T}_{\mathrm{RM}}$ to proliferate and secrete cytokines; the complete model, including all cytokine functions, best fit to the data (Fig. S2) (C) Three simulated episodes starting at different E:T ratios; log $10 \mathrm{HSV}-2$ DNA (red) and relative cytokine concentration (blue). (D,E) 1000 simulated episodes with starting E:T ratio densities randomly selected from Fig. 2d; inverse correlation of the ratio of $\mathrm{T}_{\mathrm{RM}}$ to epithelial cells within (D) the entire model grid of 15625 cells and (E) the 49 cells surrounding the first infected cell to peak viral load. 
A

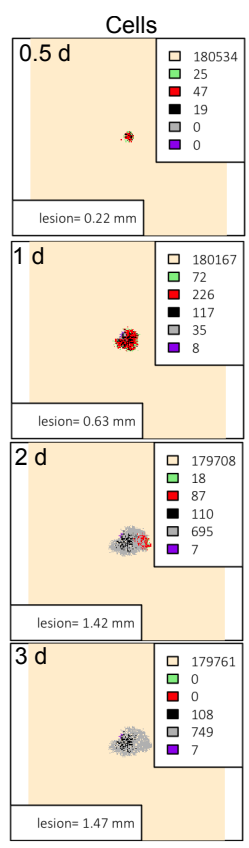

B
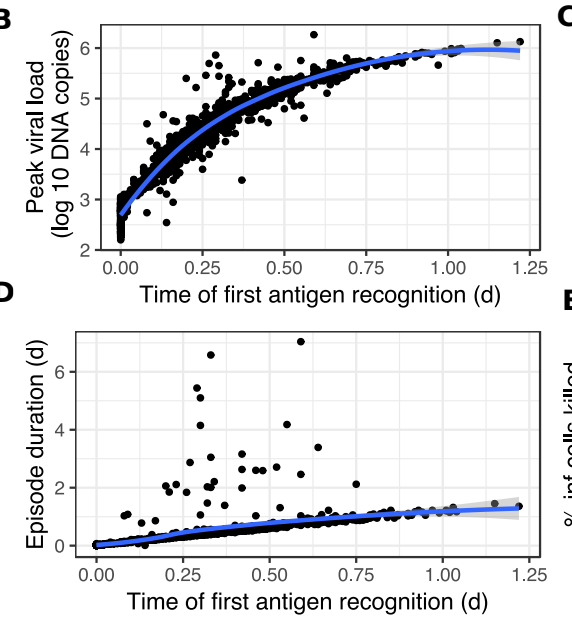
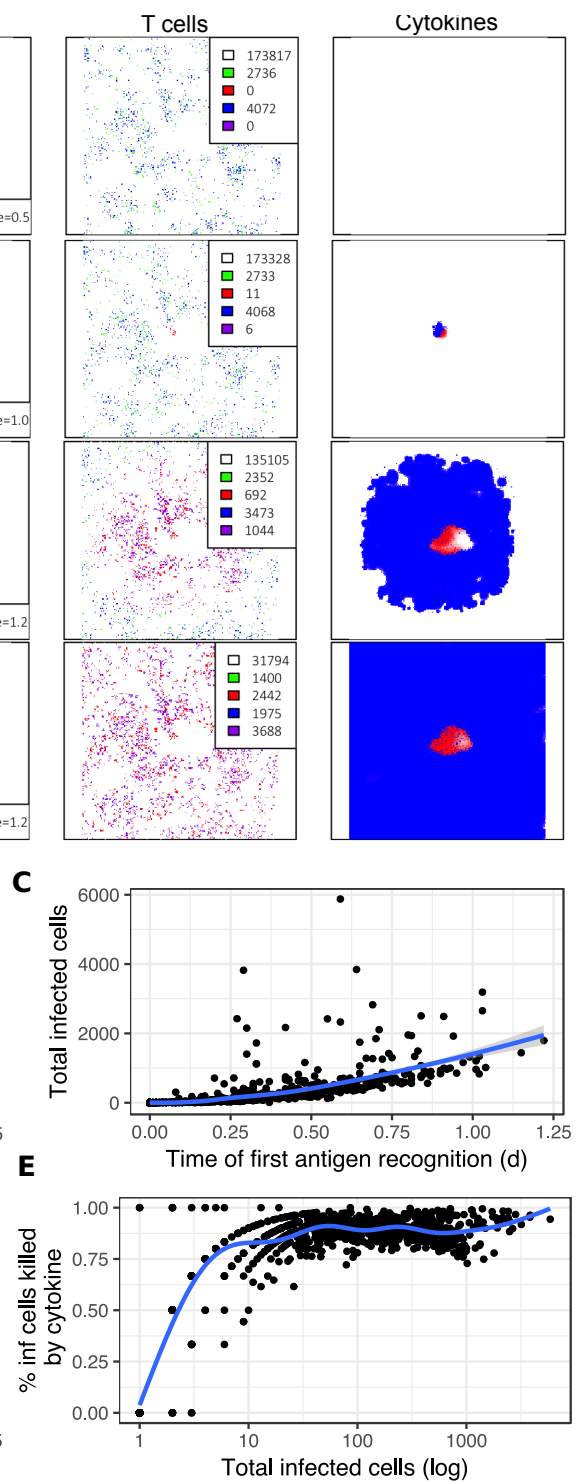

Fig. 6. Cytokine spread leading to rapid elimination of infected HSV-2 cells. (A) A simulated episode (corresponding to Movies S5 \& S6) with low $\mathrm{T}_{\mathrm{RM}}$ density leading to control of infection. Panels left to right: (1) epidermal cell dynamics, peach $=$ uninfected, green $=$ pre-productive infection, red $=$ productive infection, black $=$ virally lysed cell, purple $=\mathrm{T}_{\mathrm{RM}}$ lysed cell, grey $=$ cytokine lysed cell; (2) viral loads (HSV DNA copies) per cell: purple $=10^{2}-10^{2.25}$, blue $=10^{2.25}$. $10^{2.5}$, green $=10^{2.5}-10^{2.75}$, yellow $=10^{2.75}-10^{3}$, orange $=10^{3}-10^{3.25}$, red $=10^{3.25}-10^{3.5}$, dark red $>10^{3.5}$; (3) $\mathrm{T}_{\mathrm{RM}}$, green $=$ inactivated HSV-specific $\mathrm{T}_{\mathrm{RM}}$, red = activated HSV-specific $\mathrm{T}_{\mathrm{RM}}$, blue $=$ inactivated bystander $\mathrm{T}_{\mathrm{RM}}$, purple = activated HSV-specific $\mathrm{T}_{\mathrm{RM}}$; (4) Concentration of cytokine by color darkness; red over infected or dead cells, indicating reduced viral replication and infected cell lifespan; blue cover uninfected cells, indicating reduced viral infectivity. (B-E) 1000 simulated episodes with initial E:T ratio densities randomly selected from Fig. 2 d. Correlations of time to first $\mathrm{T}_{\mathrm{RM}}$ recognition of an infected cell with (B) $\log 10$ peak viral load, (C) total infected cells, and (D) duration. (E) Proportion of infected cells killed by cytokine effects as a function of number of total infected cells per episode. Blue lines = LOESS-smoothed lines with $95 \% \mathrm{CI}$ in grey. 


\section{Supplementary Materials:}

A
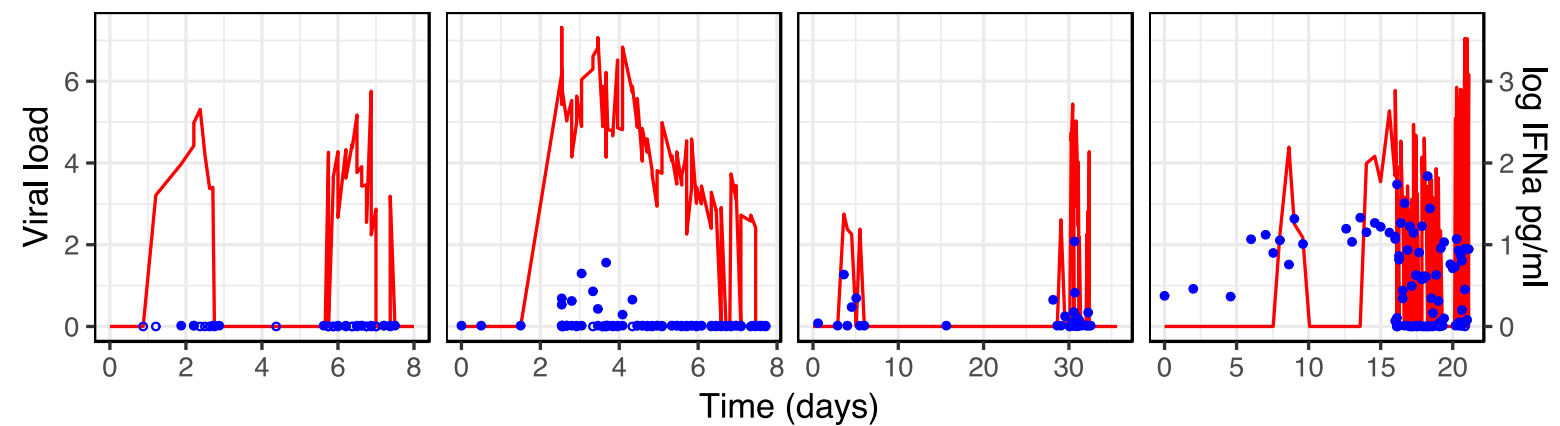

B
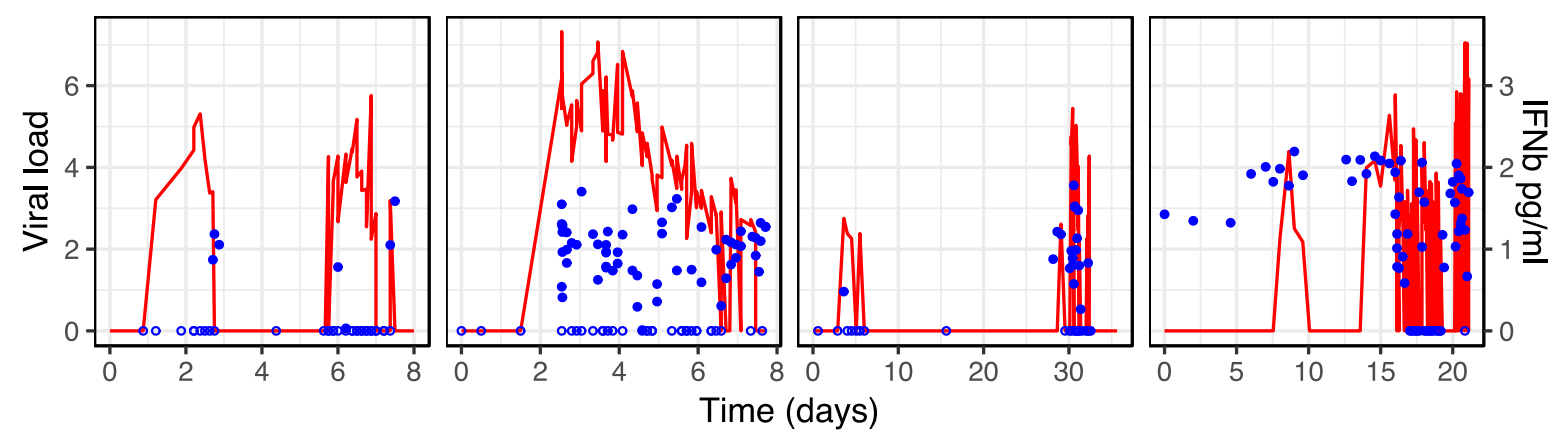

Fig. S1. No predictable surge of epithelial-derived antiviral cytokines during HSV-2 shedding episodes. Detailed cytokine kinetics from episodes in four infected persons who performed swabs every 8 hours pre-lesion detection and every 3 hours post-lesion detection; equivalent episodes aligned vertically from Figure 5a; red line = HSV-2 DNA (left y-axis). (A) IFN- $\alpha$ (blue dots) during viral expansion. (B) IFN- $\beta$ (blue dots) during viral expansion. 


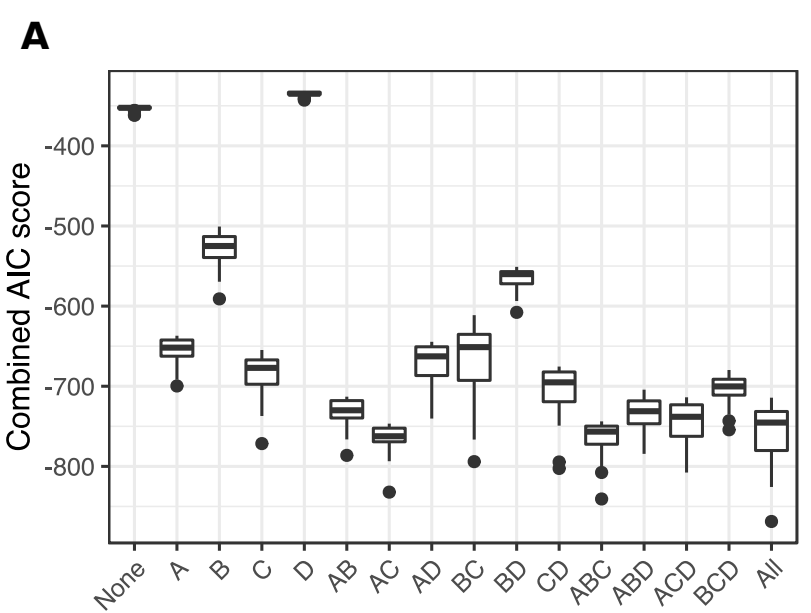

Models with different cytokine effects

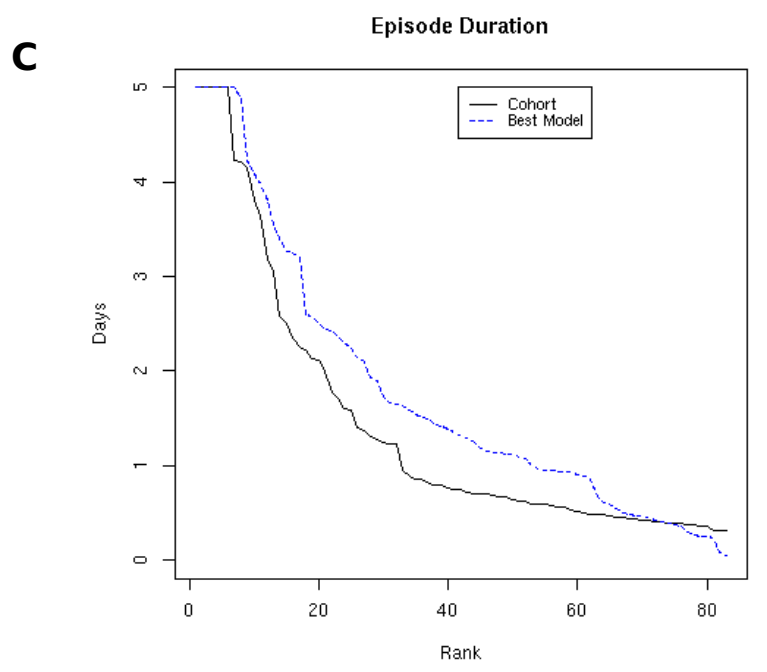

Peak Viral Load

B

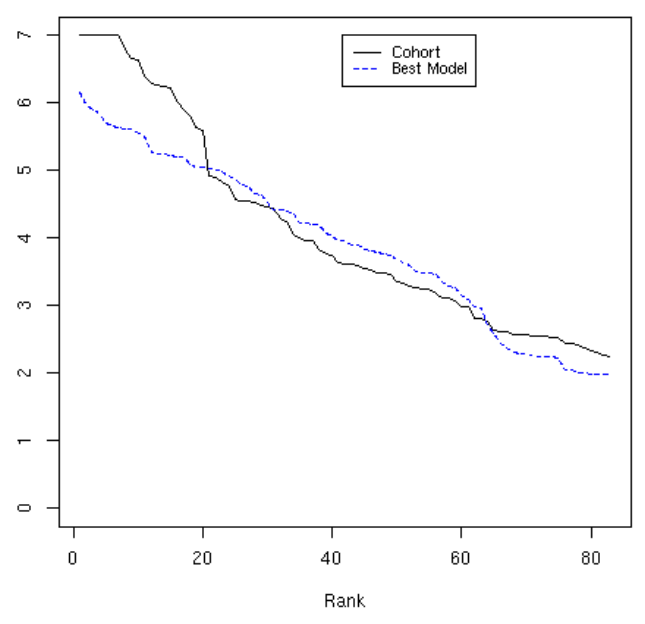

D

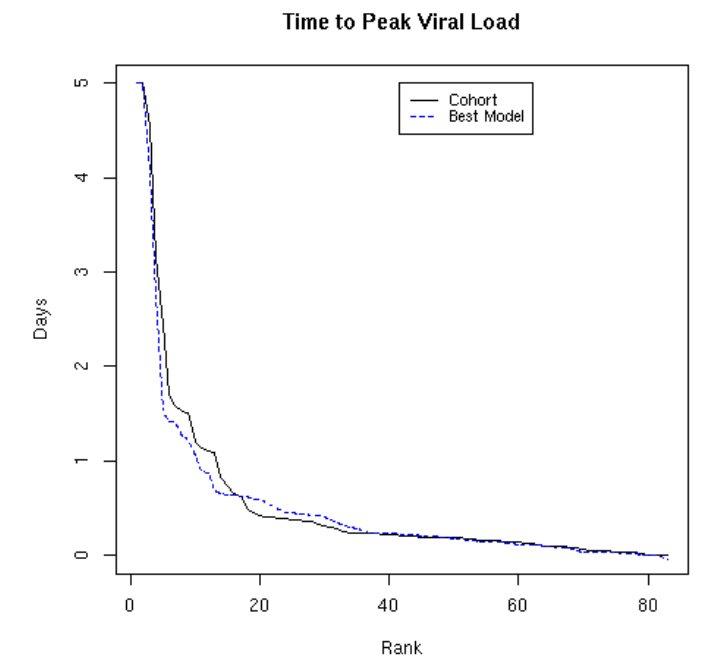

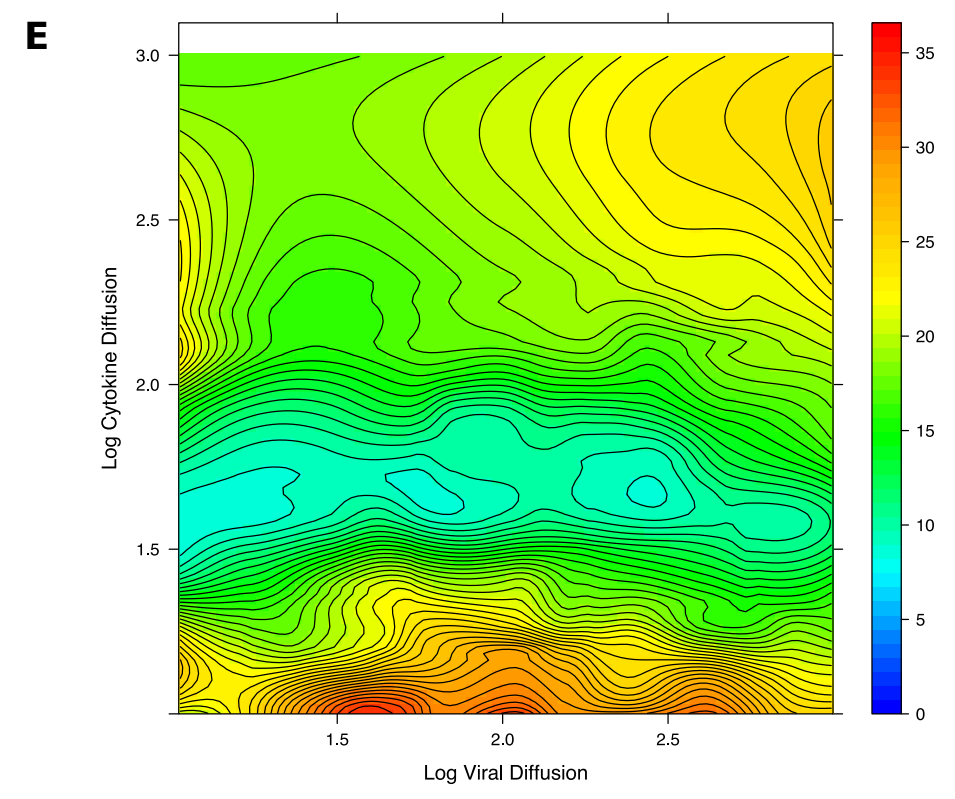


Fig. S2. A model with all possible antiviral cytokine features with optimal fit to the data. (A) Optimized AIC scores for models including and excluding various possible cytokine functions including: A, increasing infected cell death rate; $\mathrm{B}$, decreasing viral infectivity; $\mathrm{C}$, lowering viral replication rate in infected cells; and $\mathrm{D}$, activating other bystander $\mathrm{T}_{\mathrm{RM}}$; low AIC scores represent higher likelihood models; lowest AIC scores occur with the most inclusive model; boxplots $=$ interquartile ranges $(\mathrm{IQR})$ and whiskers $=$ ranges or $1.5 \mathrm{x}$ the IQR for 10 simulations of each model with 100 episodes per simulation. (B-D) Rank abundance curves of 83 episodes sorted from highest to lowest according to episode (B) peak viral loads, (C) duration and (D) time to peak viral load; black lines $=$ episode data and blue lines $=$ optimized values from the mathematical model. (E) Heat maps for two model parameter values (rate of viral diffusion and rate of cytokine diffusion) from the completely inclusive best-fitting model with range of values required for best fit to the data for cytokine diffusion rate and viral diffusion rate; color values $=$ residual sum of squares between model output and the data in B-D: blue represents best model fit. 

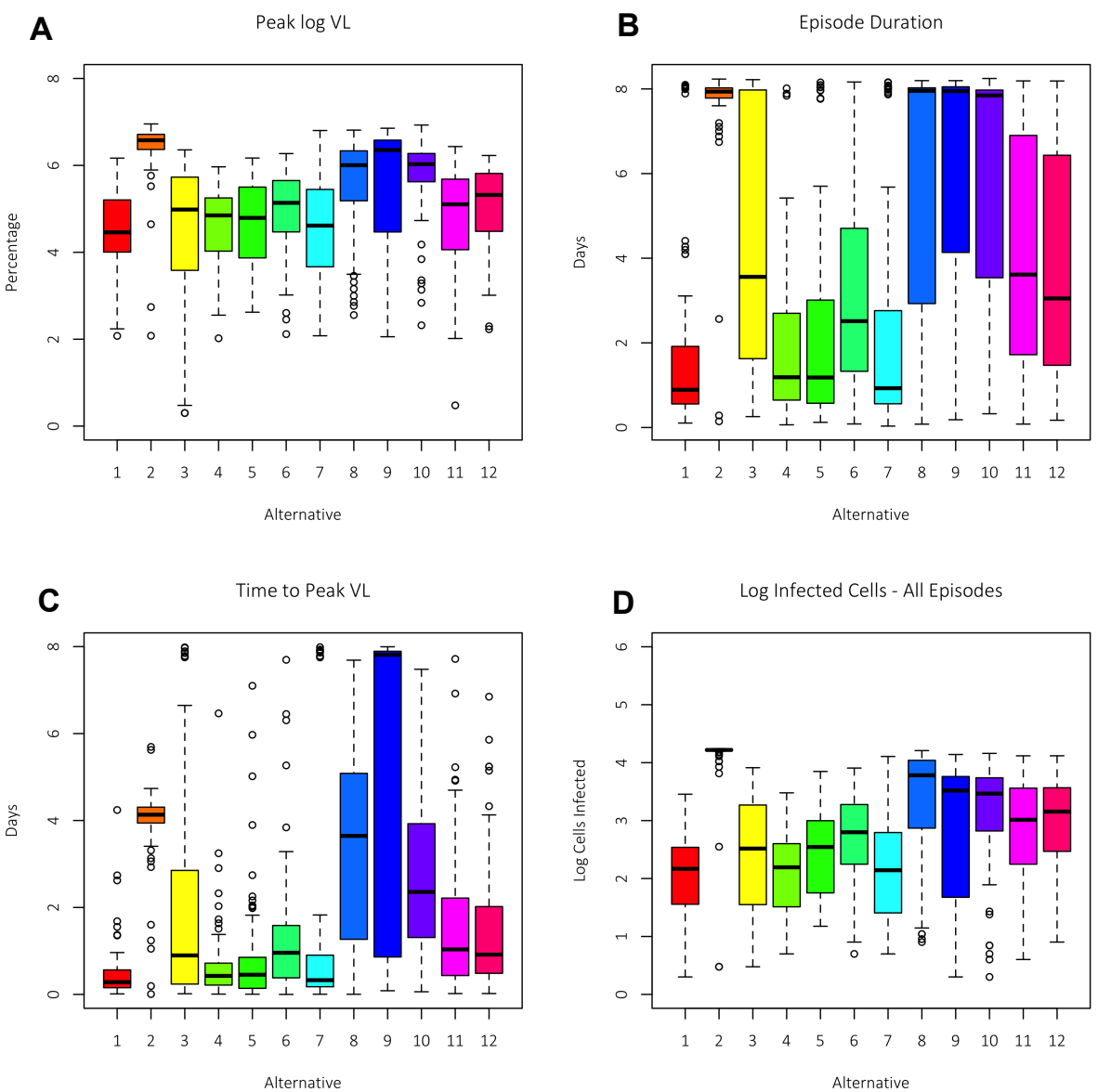

1: Baseline Best model from the 16 model comparison

2: Best model minus all cytokine effects

3: Best model minus cytokine effect on infected cell lifespan

4: Best model minus cytokine effect on viral reproduction rate

5: Best model minus cytokine effect on viral infectivity

6: Best model minus cytokine effect on TRM activation (proliferation \& cytokine production)

7: Best model minus cytokine effect on TRM activation (proliferation only)

8: Best model minus cytokine effect on TRM activation (cytokine only)

9: Best model minus cytokine effect on infected cell lifespan \& viral reproduction rate

10: Best model minus cytokine effect on infected cell lifespan, viral reproduction rate \& TRM activation (proliferation \& cytokine production)

11: Best model minus cytokine effect on infected cell lifespan, viral infectivity \& TRM activation (proliferation \& cytokine production)

12: Best model minus cytokine effect on viral infectivity, viral reproduction rate \& TRM activation (proliferation \& cytokine production

Fig. S3. Polyfunctional and individually redundant antiviral cytokine effects within the HSV-2 infection micro-environment. Episodes simulated with starting E:T ratio densities randomly selected from Fig. 2d under conditions in which cytokine effects were limited to one or more of the following mechanisms: enhancing infected cell death rate, decreasing viral replication rate in infected cells, decreasing infectivity to uninfected cells and activating local bystander $\mathrm{T}_{\mathrm{RM}}$; one thousand episodes simulated per model; model descriptions below the figure; episode severity measured according to (A) time to peak viral load, (B) $\log 10$ total number of infected cells by 5 days, (C) $\log 10$ peak viral load and (D) duration; results displayed with histograms showing median, interquartile range box, values within 1.5 of the IQR (whiskers) and individual episodes (dots); models with removal of single cytokine effects continue to allow rapid control of most episodes indicating that multiple, but not all antiviral functions are required to achieve rapid elimination of infection. 


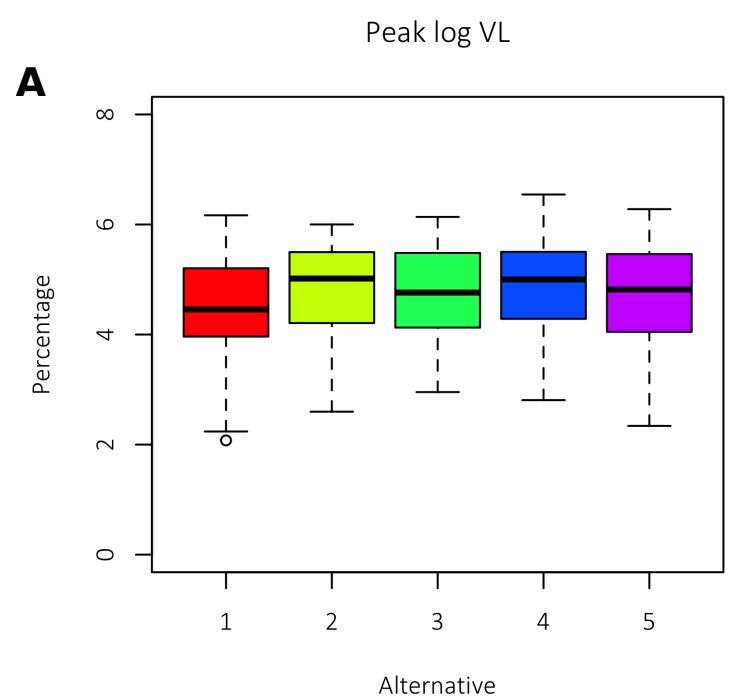

Time to Peak VL

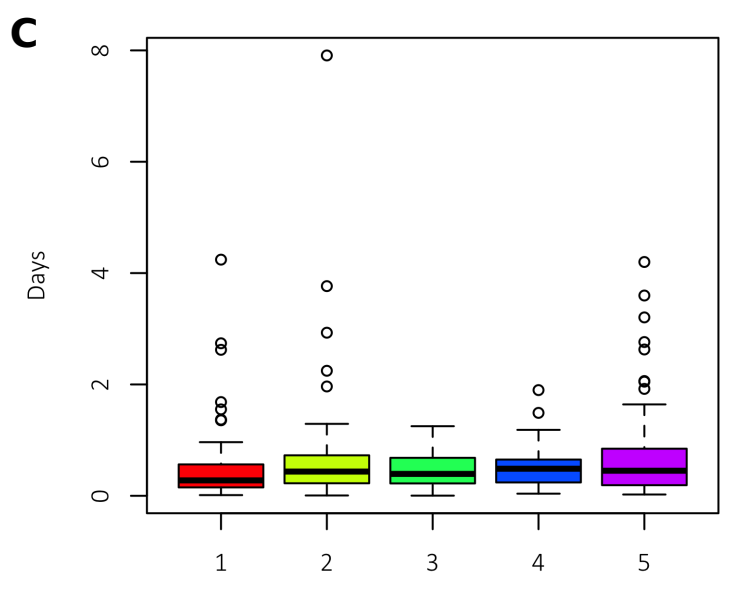

Alternative

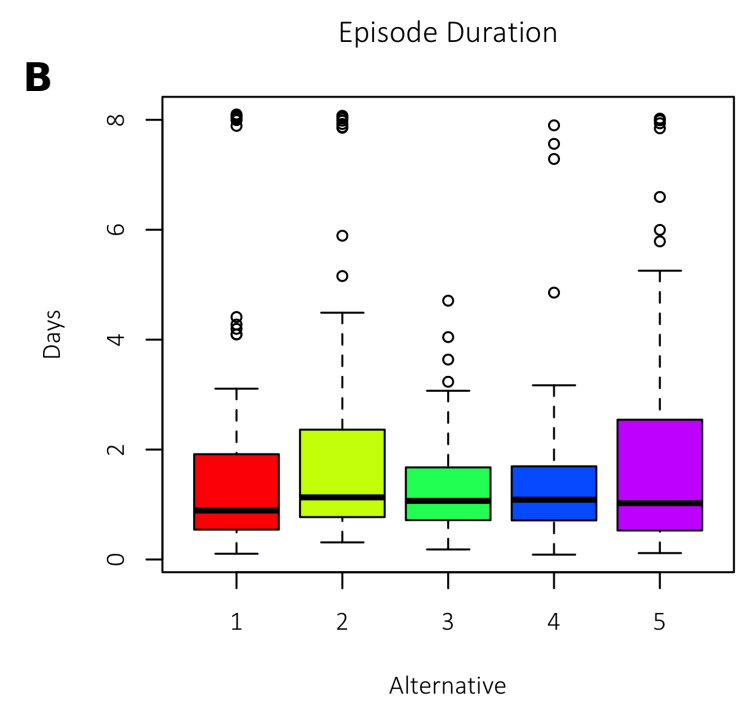

Log Infected Cells - All Episodes

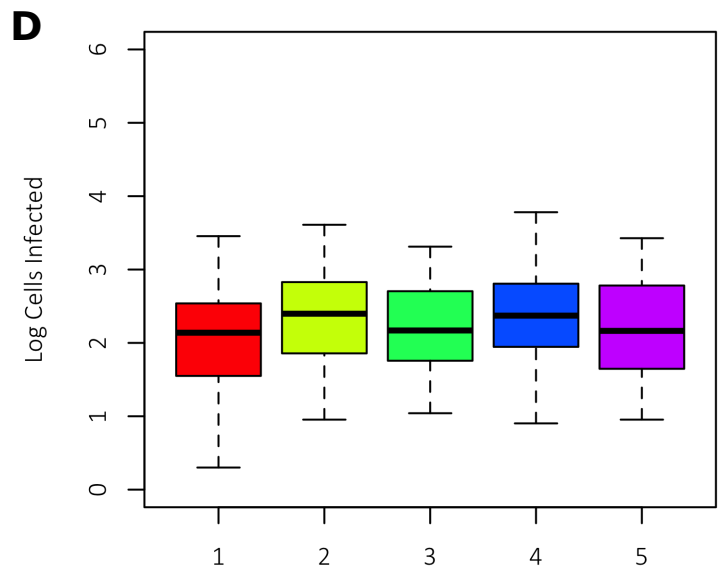

Alternative

1: Baseline Best model from the 16 model comparison

2: Best model minus Trm dendricity

3: Best model minus Trm mobility

4: Best model minus Trm dendricity \& mobility

5: Best model minus Trm mediated contact killing

Fig. S4. No requirement of trafficking, dendricity, and contact-mediated killing of $\mathbf{T}_{\mathrm{RM}}$ for elimination of HSV-2 infected cells during severe episodes. Episodes simulated with starting E:T ratio densities randomly selected from Fig. $2 d$ under conditions in which $\mathrm{T}_{\mathrm{RM}}$ dendricity and / or trafficking is absent, and that $\mathrm{T}_{\mathrm{RM}}$ contact-mediated killing is absent; one thousand episodes simulated per model; model descriptions below the figure; episode severity measured according to (A) time to peak viral load, (B) $\log 10$ total number of infected cells by 5 days, (C) $\log 10$ peak viral load and (D) duration; results displayed with histograms showing median, interquartile range box, values within 1.5 of the IQR (whiskers) and individual episodes (dots). 
Movie S1. Lack of containment of HSV-2 spread in the absence of $\mathbf{T}_{\mathrm{RM}}$. One simulated episode on a large cell field. Left panel: ulcer cell dynamics with cell counts in the legend, peach $=$ uninfected cells, green $=$ pre-productive infection, red $=$ productive infection, black $=$ virally lysed cell. Right panel: Viral loads (HSV DNA copies) per cellular region: purple $=10^{2}$ $10^{2.25}$, blue $=10^{2.25}-10^{2.5}$, green $=10^{2.5}-10^{2.75}$, yellow $=10^{2.75}-10^{3}$, orange $=10^{3}-10^{3.25}$, red $=10^{3.25}$ $10^{3.5}$, dark red $>10^{3.5}$.

Movie S2. Lack of containment of HSV-2 spread in the presence of $\mathbf{T}_{\mathrm{RM}}$ which only kill via direct lysis of infected cells. One simulated episode on a large cell field. Left panel: ulcer cell dynamics with cell counts in the legend, peach $=$ uninfected cells, green $=$ pre-productive infection, red $=$ productive infection, black $=$ virally lysed cell, purple $=\mathrm{T}_{\mathrm{RM}}$ lysed cell. Middle panel: viral loads (HSV DNA copies) per cellular region: purple $=10^{2}-10^{2.25}$, blue $=10^{2.25}-10^{2.5}$, green $=10^{2.5}-10^{2.75}$, yellow $=10^{2.75}-10^{3}$, orange $=10^{3}-10^{3.25}$, red $=10^{3.25}-10^{3.5}$, dark red $>10^{3.5}$. Right panel: tissue-resident $\mathrm{T}$ cell dynamics with cell counts in the legend, green = inactivated HSVspecific $\mathrm{T}_{\mathrm{RM}}$, red $=$ activated HSV-specific $\mathrm{T}_{\mathrm{RM}}$, blue $=$ inactivated bystander $\mathrm{T}_{\mathrm{RM}}$, purple $=$ activated bystander $\mathrm{T}_{\mathrm{RM}}$.

Movie S3. Lack of containment of HSV-2 spread in the presence of $\mathbf{T}_{\mathrm{RM}}$ which only kill via direct lysis of infected cells. One simulated episode on a small cell field. Left panel: ulcer cell dynamics with cell counts in the legend, peach $=$ uninfected cells, green $=$ pre-productive infection, red $=$ productive infection, black $=$ virally lysed cell, purple $=\mathrm{T}_{\mathrm{RM}}$ lysed cell . Middle panel: viral loads (HSV DNA copies) per cellular region: purple $=10^{2}-10^{2.25}$, blue $=10^{2.25}-10^{2.5}$, green $=10^{2.5}-10^{2.75}$, yellow $=10^{2.75}-10^{3}$, orange $=10^{3}-10^{3.25}$, red $=10^{3.25}-10^{3.5}$, dark red $>10^{3.5}$. Right panel: tissue-resident $\mathrm{T}$ cell dynamics with cell counts in the legend, green = inactivated HSVspecific $\mathrm{T}_{\mathrm{RM}}$, red = activated HSV-specific $\mathrm{T}_{\mathrm{RM}}$, blue $=$ inactivated bystander $\mathrm{T}_{\mathrm{RM}}$, purple $=$ activated bystander $\mathrm{T}_{\mathrm{RM}}$.

Movie S4. Rapid containment of HSV-2 spread in the presence of $T_{R M}$ antiviral cytokine secretion and high $\mathbf{T}_{\mathbf{R M}}$ density. Four simulated episodes with high initial $\mathrm{T}_{\mathrm{RM}}$ density leading to rapid control of infection on a small cell field. Left upper panel: ulcer cell dynamics with cell counts in the legend, peach= uninfected cells, green = pre-productive infection, red = productive infection, black $=$ virally lysed cell, purple $=\mathrm{T}_{\mathrm{RM}}$ lysed cell, grey $=$ cytokine lysed cell. Right upper panel: viral loads (HSV DNA copies) per cellular region: purple $=10^{2}-10^{2.25}$, blue $=10^{2.25}-10^{2.5}$, green $=10^{2.5}-10^{2.75}$, yellow $=10^{2.75}-10^{3}$, orange $=10^{3}-10^{3.25}$, red $=10^{3.25}-10^{3.5}$, dark red $>10^{3.5}$. Left lower panel: tissue-resident $\mathrm{T}$ cell dynamics with cell counts in the legend, green $=$ inactivated HSV-specific $\mathrm{T}_{\mathrm{RM}}$, red = activated HSV-specific $\mathrm{T}_{\mathrm{RM}}$, blue $=$ inactivated bystander $\mathrm{T}_{\mathrm{RM}}$, purple $=$ activated bystander $\mathrm{T}_{\mathrm{RM}}$. Right lower panel = concentration of cytokine indicated by darkness of color; red is over infected cells or dead cells, indicating limitation of viral replication and infected cell lifespan; blue is over uninfected cells, indicating limitation of new infection.

\section{Movie S5. Containment of HSV-2 spread in the presence of $\mathbf{T}_{\mathrm{RM}}$ antiviral cytokine secretion and low $\mathbf{T}_{\mathbf{R M}}$ density. Four simulated episodes on a small cell field with low initial $\mathrm{T}_{\mathrm{RM}}$ density leading to slower control of infection. Left upper panel: ulcer cell dynamics with cell counts in the legend, peach= uninfected cells, green = pre-productive infection, $\mathrm{red}=$ productive infection, black = virally lysed cell, purple $=\mathrm{T}_{\mathrm{RM}}$ lysed cell, grey $=$ cytokine lysed cell . Right upper panel: viral loads (HSV DNA copies) per cellular region: purple $=10^{2}-10^{2.25}$,}


blue $=10^{2.25}-10^{2.5}$, green $=10^{2.5}-10^{2.75}$, yellow $=10^{2.75}-10^{3}$, orange $=10^{3}-10^{3.25}$, red $=10^{3.25}-10^{3.5}$, dark $\mathrm{red}>10^{3.5}$. Left lower panel: tissue-resident $\mathrm{T}$ cell dynamics with cell counts in the legend, green = inactivated HSV-specific $\mathrm{T}_{\mathrm{RM}}$, red $=$ activated HSV-specific $\mathrm{T}_{\mathrm{RM}}$, blue $=$ inactivated bystander $\mathrm{T}_{\mathrm{RM}}$, purple $=$ activated bystander $\mathrm{T}_{\mathrm{RM}}$. Right lower panel $=$ concentration of cytokine indicated by darkness of color; red is over infected cells or dead cells, indicating limitation of viral replication and infected cell lifespan; blue is over uninfected cells, indicating limitation of new infection.

Movie S6. Containment of HSV-2 spread in the presence of $\mathbf{T}_{\mathrm{RM}}$ antiviral cytokine secretion and low $\mathbf{T}_{\mathbf{R M}}$ density. A simulated episode on a large cell field with low initial $\mathrm{T}_{\mathrm{RM}}$ density leading to slower control of infection. Left upper panel: ulcer cell dynamics with cell counts in the legend, peach= uninfected cells, green = pre-productive infection, red = productive infection, black $=$ virally lysed cell, purple $=\mathrm{T}_{\mathrm{RM}}$ lysed cell, grey $=$ cytokine lysed cell. Right upper panel: viral loads (HSV DNA copies) per cellular region: purple $=10^{2}-10^{2.25}$, blue $=10^{2.25}-10^{2.5}$, green $=10^{2.5}-10^{2.75}$, yellow $=10^{2.75}-10^{3}$, orange $=10^{3}-10^{3.25}$, red $=10^{3.25}-10^{3.5}$, dark red $>10^{3.5}$. Left lower panel: tissue-resident $\mathrm{T}$ cell dynamics with cell counts in the legend, green $=$ inactivated $\mathrm{HSV}$-specific $\mathrm{T}_{\mathrm{RM}}$, red $=$ activated $\mathrm{HSV}$-specific $\mathrm{T}_{\mathrm{RM}}$, blue $=$ inactivated bystander $\mathrm{T}_{\mathrm{RM}}$, purple $=$ activated bystander $\mathrm{T}_{\mathrm{RM}}$. Right lower panel = concentration of cytokine indicated by darkness of color; red is over infected cells or dead cells, indicating limitation of viral replication and infected cell lifespan; blue is over uninfected cells, indicating limitation of new infection.

\section{Movie S7. Slower containment of HSV-2 spread in the presence of $\mathbf{T}_{\mathrm{RM}}$ antiviral cytokine secretion which only prevents infectivity. Four simulated episodes. Left upper panel: ulcer cell dynamics with cell counts in the legend, peach $=$ uninfected cells, green $=$ pre-productive infection, red $=$ productive infection, black $=$ virally lysed cell, purple $=\mathrm{T}_{\mathrm{RM}}$ lysed cell, grey $=$ cytokine lysed cell. Right upper panel: viral loads (HSV DNA copies) per cellular region: purple $=10^{2}-10^{2.25}$, blue $=10^{2.25}-10^{2.5}$, green $=10^{2.5}-10^{2.75}$, yellow $=10^{2.75}-10^{3}$, orange $=10^{3}-10^{3.25}$, red $=10^{3.25}-10^{3.5}$, dark red $>10^{3.5}$. Left lower panel: tissue-resident $\mathrm{T}$ cell dynamics with cell counts in the legend, green = inactivated HSV-specific $\mathrm{T}_{\mathrm{RM}}$, red = activated HSV-specific $\mathrm{T}_{\mathrm{RM}}$, blue $=$ inactivated bystander $\mathrm{T}_{\mathrm{RM}}$, purple $=$ activated bystander $\mathrm{T}_{\mathrm{RM}}$. Right lower panel $=$ concentration of cytokine indicated by darkness of color; blue is over uninfected cells, indicating limitation of new infection.}

\section{Movie S8. Slightly protracted containment of HSV-2 spread in the presence of $\mathbf{T}_{\mathbf{R M}}$ antiviral cytokine secretion which only limit lifespan of infected cells. Four simulated} episodes. Left upper panel: ulcer cell dynamics with cell counts in the legend, peach= uninfected cells, green $=$ pre-productive infection, red $=$ productive infection, black $=$ virally lysed cell, purple $=\mathrm{T}_{\mathrm{RM}}$ lysed cell, grey $=$ cytokine lysed cell. Right upper panel: viral loads (HSV DNA copies) per cellular region: purple $=10^{2}-10^{2.25}$, blue $=10^{2.25}-10^{2.5}$, green $=10^{2.5}-10^{2.75}$, yellow $=10^{2.75}$ $10^{3}$, orange $=10^{3}-10^{3.25}$, red $=10^{3.25}-10^{3.5}$, dark red $>10^{3.5}$. Left lower panel: tissue-resident $\mathrm{T}$ cell dynamics with cell counts in the legend, green = inactivated HSV-specific $\mathrm{T}_{\mathrm{RM}}$, red = activated HSV-specific $\mathrm{T}_{\mathrm{RM}}$, blue $=$ inactivated bystander $\mathrm{T}_{\mathrm{RM}}$, purple $=$ activated bystander $\mathrm{T}_{\mathrm{RM}}$. Right lower panel = concentration of cytokine indicated by darkness of color; red is over infected cells or dead cells, indicating limitation of viral replication and infected cell lifespan. 